\title{
Dynamic structure factor and dielectric function of silicon for finite momentum transfer: Inelastic x-ray scattering experiments and $a b$ initio calculations
}

\author{
Hans-Christian Weissker, ${ }^{1,2,3}$ Jorge Serrano, ${ }^{4,5}$ Simo Huotari, ${ }^{4}$ Eleonora Luppi, $, 2,3$ Marco Cazzaniga,, 3 Fabien Bruneval, ${ }^{7,3}$ \\ Francesco Sottile, ${ }^{2,3}$ Giulio Monaco, ${ }^{4}$ Valerio Olevano, ${ }^{8,3}$ and Lucia Reining ${ }^{2,3}$ \\ ${ }^{1}$ LPICM, Ecole Polytechnique, CNRS, F-91128 Palaiseau, France \\ ${ }^{2}$ Laboratoire des Solides Irradiés, Ecole Polytechnique, CNRS-CEA/DSM, F-91128 Palaiseau, France \\ ${ }^{3}$ European Theoretical Spectroscopy Facility (ETSF) \\ ${ }^{4}$ European Synchrotron Radiation Facility (ESRF), 6 rue Jules Horowitz, BP 220, 38042 Grenoble Cedex 9, France \\ 5 ICREA, Departament de Fisica Aplicada, EPSC, Universitat Politècnica de Catalunya, Avenida Canal Olimpic 15, \\ 08860 Castelldefels, Spain \\ ${ }^{6}$ Università degli Studi di Milano, I-20133 Milan, Italy \\ ${ }^{7}$ CEA, DEN, Service de Recherches de Métallurgie Physique, F-91191 Gif-sur-Yvette, France \\ ${ }^{8}$ Institut Néel, CNRS and UJF, F-38042 Grenoble, France
}

(Received 12 October 2009; revised manuscript received 12 December 2009; published 3 February 2010)

\begin{abstract}
We present a detailed investigation of the dynamic structure factor $S(\mathbf{Q}, \omega)$ as well as of the dielectric function $\varepsilon_{\mathrm{M}}(\mathbf{Q}, \omega)$ of the prototypical semiconductor silicon for finite momentum transfer, combining inelastic $\mathrm{X}$-ray scattering measurements and $a b$ initio calculations. We show that, in contrast to optical spectra, for finite momentum transfer, time-dependent density-functional theory in adiabatic local-density approximation (TDLDA) together with the inclusion of lifetime effects in a modified independent-particle polarizability $\chi^{0, \mathrm{LT}}$ describes the physics of valence excitations with high precision. This applies to the dynamic structure factor as well as to the dielectric function, which demonstrates that TDLDA contains the short-range many-body effects that are crucial for a correct description of $\varepsilon_{M}(\mathbf{Q}, \omega)$ in silicon at finite momentum transfer. The form of a nonlocal and energy-dependent exchange-correlation kernel is presented which provides the inclusion of the lifetime effects using the true independent-particle polarizability $\chi^{0}$. The description of the silicon $L_{2,3}$ absorption edge has been possible by including the outer core electrons $2 s$ and $2 p$ in the valence electrons of the pseudopotential. The energy of the edge is underestimated but a scissor shift of the respective states by the self-energy correction for these states yields good agreement with experiment. Short-range crystal local-field effects and exchange-correlation effects become important with increasing momentum transfer. The inclusion of crystal local-field effects in the random-phase approximation is able to describe the anisotropy of the response well. Our results demonstrate the quantitative predictive power of the first-principles description.
\end{abstract}

DOI: 10.1103/PhysRevB.81.085104

PACS number(s): 71.10.-w, 71.15.Dx, 78.70.Ck

\section{INTRODUCTION}

Knowledge of the electronic structure and response of semiconductor materials is crucial for the understanding of their properties. Experimental and theoretical approaches targeting electronic excitations have considerably advanced over the last years, deepening the understanding of the involved quantities and processes. The density of states can be probed approximately by direct and inverse photoemission experiments with varying degrees of bulk sensitivity due to tunable photon energies. Band structures can be measured by high-resolution angle-resolved photoemission. Both quantities are related to one-particle excitations and in general well described by $a b$ initio band-structure calculations, especially when many-body effects are properly included through the evaluation of self-energy contributions, mostly done within Hedin's $G W$ approximation. ${ }^{1}$

By contrast, a precise description of optical absorption [essentially given by the imaginary part of the dielectric function $\operatorname{Im} \varepsilon_{M}(\mathbf{Q}, \omega)$ for vanishing momentum transfer] had long been prohibitive in this framework because it presents a two-particle problem. Eventually, semiempirical ${ }^{2}$ and, later, $a b$ initio $^{3,4}$ calculations based on the solution of the BetheSalpeter equation of many-body perturbation theory ${ }^{4}$ have shown that the inclusion of the electron-hole interaction is both necessary and sufficient in order to obtain very good agreement between measured and calculated absorption spectra.

An alternative approach to electronic excitations is presented by time-dependent density-functional theory (TDDFT). ${ }^{5}$ The central quantity here is the time-dependent electron density. In the framework of linear response, ${ }^{6}$ the electronic spectra can be determined using certain approximations of the exchange-correlation kernel $f_{\mathrm{xc}}$ which represents the exchange and correlation effects in the electronic response of the system. A kernel has been developed recently which is able to reproduce the very good Bethe-Salpeter results. ${ }^{7-12}$ However, the calculations using this kernel remain computationally very demanding. Therefore there is a practical interest in simpler approximations which can be applied more easily. The simplest approximation beyond the random-phase approximation (RPA) is the adiabatic localdensity approximation (ALDA), in connection with TDDFT also referred to as time-dependent local-density approximation (TDLDA). ${ }^{6}$ Unfortunately it turns out that the situation of optical absorption in bulk materials is not well described by the ALDA which, being a local approximation, fares best for the description of short-range effects. The results for bulk 
semiconductors as calculated in TDLDA differ only slightly from those obtained in the RPA, and they do not reproduce experiment. ${ }^{4}$ It is known, however, that the analytic form of $f_{\mathrm{xc}}$ exhibits an asymptotic $1 / r$ behavior. ${ }^{7,13,14}$ It has been shown that this long-range part of the kernel is able to mimic the electron-hole interaction. . $^{7} 15,16$

By contrast, the plasmon structures in the dynamic structure factor $S(\mathbf{Q}, \omega) \propto-\operatorname{Im} 1 / \varepsilon_{\mathrm{M}}(\mathbf{Q}, \omega)$ for vanishing momentum transfer $(\mathbf{Q} \rightarrow 0)$ are often already reasonably well reproduced by the comparably simple calculations in TDLDA. ${ }^{6}$ This has been explained ${ }^{4,17}$ by the fact that the long-range contribution missing in the exchange-correlation kernel of the TDLDA is less important in the electron energy-loss function $-\operatorname{Im} 1 / \varepsilon_{M}(\mathbf{Q}, \omega)$ and, likewise, in the dynamic structure factor than in the absorption spectrum.

The general finding for vanishing momentum transfer is that the absorption spectra, being determined by interband transitions, are not well represented by the TDLDA, whereas the collective plasmon excitation is well described. This raises the question as to how well the spectra are described for finite momentum transfer. Successful TDLDA calculations of $S(\mathbf{Q}, \omega) \propto-\operatorname{Im} 1 / \varepsilon_{\mathrm{M}}(\mathbf{Q}, \omega)$ have indeed been reported for finite $\mathbf{Q},{ }^{18-28}$ indicating good agreement with experiment for the dynamic structure factor. However, for the dielectric function $\varepsilon_{\mathrm{M}}(\boldsymbol{Q}, \omega)$ itself, no systematic comparison between theory and experiment existed up to now at finite momentum transfer, to the best of our knowledge. The analogy with optical absorption suggested that the results might be poor.

For the dynamic structure factor $S(\mathbf{Q}, \omega)$, previous measurements ${ }^{29}$ and calculations ${ }^{22,29}$ of silicon had explained the main features. However, discrepancies seemed to indicate the need for more sophisticated many-body calculations. We have recently shown ${ }^{30}$ that at least as far as valence excitations in silicon are concerned, these discrepancies have been resolved. The agreement between the experiment and the TDLDA results turns out to be excellent not only for the electron energy-loss function and the dynamic structure factor but also for the dielectric function $\varepsilon_{\mathrm{M}}(\mathbf{Q}, \omega)$ that we obtain from the measured $S(\mathbf{Q}, \omega)$ by means of the KramersKronig (KK) relations. This demonstrates that TDLDA contains the short-range many-body effects that are crucial for a correct description of $\varepsilon_{\mathrm{M}}(\mathbf{Q}, \omega)$ in silicon at finite momentum transfer.

However, we observe that the TDLDA does not include effects due to the lifetimes of electrons and holes. The inclusion of these has been shown to be necessary to diminish spurious structures which otherwise appear in the spectra. ${ }^{30}$ Nonetheless, this inclusion of lifetime effects using a modified independent-particle polarizability $\chi^{0, \mathrm{LT}}$ is not exact in the framework of linear-response TDDFT where all manybody effects in the response are represented by the exchangecorrelation kernel $f_{\mathrm{xc}}$ and the Coulomb interaction $v$. However, starting from the good result of TDLDA with added lifetime effects in the $\chi^{0, \mathrm{LT}}$, the problem can be rewritten in order to obtain the form of a nonlocal and energy-dependent kernel $f_{\mathrm{xc}}^{\mathrm{LT}}$ which produces the same effect with the true independent-particle polarizability $\chi^{0}$.

The anisotropy of the response can be characterized by the difference $S(\mathbf{Q}, \omega)\|[111]-S(\mathbf{Q}, \omega)\|[100]$ for fixed modulus of $\mathbf{Q}$. The resulting peak-valley structure has been related to plasmon Fano resonances by Schülke et al. ${ }^{29}$ who calculated the difference using the two-plasmon-band model obtaining good qualitative agreement with the measurements. ${ }^{29}$ We show that the RPA is able to reproduce the anisotropy precisely, while neglecting the crystal localfield effects (CLFE) results in large deviations. The TDLDA kernel has little effect on the anisotropy.

Like most calculations of the electronic response, our calculations use the pseudopotential approximation. For silicon, the usual core-valence partition treats the $3 s$ and $3 p$ electrons as valence electrons while the rest is represented by the pseudopotential. In order to describe the silicon $L_{2,3}$ absorption edge, we include the lower-lying $2 s$ and $2 p$ electrons in the valence. These calculations, being numerically much more demanding than the use of the standard pseudopotential with four valence electrons, show that we can describe the absorption edge in the framework of our TDLDA pseudopotential calculation, although its energy is underestimated.

The present paper is structured as follows. We review the basic physics in Sec. II after which we describe the experiment in Sec. III and the details of the calculations in Sec. VI. In particular, the inclusion of lifetime effects in the modified independent-particle polarizability $\chi^{0, \mathrm{LT}}$ is discussed in Sec. IV $\mathrm{B}$, their inclusion in the exchange-correlation kernel is treated in Sec. IV C. In Sec. V we describe the results for the dynamic structure factor, followed by those reporting the dielectric function. Subsequently we discuss the influence of crystal local-field effects and the description of the silicon $L_{2,3}$ edge by the TDLDA pseudopotential calculation.

\section{BASIC RELATIONS}

In inelastic $\mathrm{x}$-ray scattering (IXS), 31 incoming photons with momentum $\hbar \mathbf{k}_{i}$ and energy $\hbar \omega_{i}$ are scattered by the sample. During the scattering process, they transfer a momentum $\hbar \mathbf{Q}=\hbar \mathbf{k}_{i}-\hbar \mathbf{k}_{f}$ and an energy $\hbar \omega=\hbar \omega_{i}-\hbar \omega_{f}$ to the sample, where $\hbar \mathbf{k}_{f}$ and $\hbar \omega_{f}$ are the momentum and energy after the scattering process. The experiment measures the double-differential cross section which is given by the removal rate of photons from the incoming beam due to scattering into a solid angle range $[\Omega, \Omega+\mathrm{d} \Omega]$ and an energytransfer range $[\hbar \omega, \hbar \omega+\hbar \mathrm{d} \omega]$. It can be written as a product $^{32}$

$$
\frac{d^{2} \sigma}{d \Omega d \omega}=\left(\frac{d \sigma}{d \Omega}\right) \underset{\mathrm{Th}}{S} S(\mathbf{Q}, \omega),
$$

where the Thomson cross section

$$
\left(\frac{d \sigma}{d \Omega}\right)_{\mathrm{Th}}=r_{0}^{2}\left(\overrightarrow{\boldsymbol{\epsilon}}_{i} \cdot \overrightarrow{\boldsymbol{\epsilon}}_{f}\right)^{2}\left(\frac{\omega_{f}}{\omega_{i}}\right)
$$

does not contain material-specific quantities. $\overrightarrow{\boldsymbol{\epsilon}}_{i}$ and $\overrightarrow{\boldsymbol{\epsilon}}_{f}$ are the respective polarization vectors. The information about the material's response is contained in the dynamic structure factor $S(\mathbf{Q}, \omega)$ which is the Fourier transform in time and space of the ground-state expectation value of the time-dependent density-density correlation operator. ${ }^{31}$ Using the fluctuationdissipation theorem, ${ }^{33} S(\mathbf{Q}, \omega)$ can be written in terms of the dielectric function $\varepsilon_{\mathrm{M}}(\mathbf{Q}, \omega) \mathrm{as}^{31}$ 


$$
S(\mathbf{Q}, \omega)=-\frac{\hbar Q^{2}}{4 \pi^{2} e^{2} n} \operatorname{Im} \frac{1}{\varepsilon_{\mathrm{M}}(\mathbf{Q}, \omega)},
$$

$n$ being the electron density. The dielectric function is given $^{34,35}$ by the inverse of the diagonal element of the inverse dielectric matrix,

$$
\varepsilon_{\mathrm{M}}(\mathbf{Q}, \omega)=\frac{1}{\varepsilon^{-1}(\mathbf{q}, \omega)_{\mathbf{G G}}},
$$

where we introduce $\mathbf{Q}=\mathbf{q}+\mathbf{G}$ with $\mathbf{q} \in \mathrm{BZ}$. The dielectric matrix is calculated in different approximations which will be described below.

The dynamic structure factor obeys the sum rule ${ }^{31}$

$$
\int_{0}^{\infty} S(\mathbf{Q}, \omega) \omega d \omega=\hbar^{2} Q^{2} / 2 m
$$

which can be used to obtain the normalization of the experimental spectra.

Equation (3) shows that the dynamic structure factor is proportional to the electron energy-loss function $-\operatorname{Im} 1 / \varepsilon_{\mathrm{M}}(\mathbf{Q}, \omega)$ measured by angle-resolved electron energy-loss spectroscopy (EELS). The quantity probed by the two experimental methods is, therefore, the same as far as the theoretical description is concerned. IXS results profit from the factor $Q^{2}$ in Eq. (3) for larger momentum transfers where EELS results are plagued by multiple scattering. For small momentum transfers, the absence of the $Q^{2}$ factor benefits the EELS results. Moreover, EELS is far more surface sensitive than IXS. Measuring the same quantity, the two experimental techniques are hence complementary.

\section{MEASUREMENTS}

We have performed nonresonant inelastic x-ray scattering measurements of $S(\mathbf{Q}, \omega)$ for $\mathbf{Q}$ between 0.27 and 2.4 a.u. along the [100] and [111] directions at room temperature. The measurements were carried out at ID16 (Refs. 36 and 37) of the European Synchrotron Radiation Facility (ESRF) using monochromatic $x$ rays with energies in the range 7.9$8.0 \mathrm{keV}$. The radiation from the undulator source was monochromated by a cryogenically cooled $\mathrm{Si}(111)$ double-crystal monochromator to a bandwidth of $0.9 \mathrm{eV}$, and for highresolution measurements with an additional $\mathrm{Si}(440)$ channelcut postmonochromator to a bandwidth of $0.15 \mathrm{eV}$. A spherically bent $\mathrm{Si}(444)$ analyzer crystal with a bending radius of 1 $\mathrm{m}$ and a diameter of $100 \mathrm{~mm}$ was used. The momentum transfer resolution was $\Delta q \approx 0.2$ a.u., achieved by reducing the active size of the analyzer crystal. As sample, a silicon wafer of $1 \mathrm{~cm}^{2}$ surface and $65 \mu \mathrm{m}$ thickness oriented with the surface normal along the [110] direction was used. The spectra were taken in transmission geometry for the directions $\mathbf{Q} \|[111]$ and $\mathbf{Q} \|[100]$. Most of the spectra were taken using an energy resolution of $1.1 \mathrm{eV}$ full width at half maximum (FWHM). No additional spectral features were observed when the energy resolution was increased to $0.2 \mathrm{eV}$. The only spectrum measured with a resolution of $0.2 \mathrm{eV}$ is that for $\mathbf{Q}=0.53$ a.u. along [100].

The background was subtracted using an estimate from the signal measured for negative energy loss. The spectra were first normalized to the incoming photon flux. The contribution from the elastic line was then removed by fitting the high-energy tail of the elastic line by an exponential which was then subtracted. The spectra thus obtained are reliable above $\sim 3 \mathrm{eV}$. Due to the presence of the $L$ absorption edge at $99.8 \mathrm{eV}$, the spectra were extrapolated from $98 \mathrm{eV}$ up to $200 \mathrm{eV}$ with an exponential tail. The sum rule Eq. (5) was then applied to obtain the dynamic structure factor $S(\mathbf{Q}, \omega)$ on an absolute scale. ${ }^{19}$ This procedure yielded reliable spectra except for the spectra of $\mathbf{Q}=0.27$ a.u. for which the rescaling had to be applied which is treated below in Sec. V C for the analysis by means of the Kramers-Kronig relations. The measurements were done in the so-called inverse geometry, where the intensity for a fixed scattered-photon energy is observed, and the incident-photon energy is scanned to create the desired energy-transfer range. In this type of experiment and when the sample is a single crystal, unwanted Bragg diffraction conditions within the sample may occur at certain well-defined incident-photon energies. When such diffraction takes place, it may be observed either as a sharp peak or a drop of intensity recorded by the analyzer crystal. These artifacts in the spectra are colloquially called "glitches" and may pose a problem for single-crystal experiments with large energy ranges to be scanned. A few such glitches were also observed in the present experiment, most importantly in the spectra with $\mathbf{Q} \|[100]$; for $\mathbf{Q}=2.12$ a.u. at 23 and $84 \mathrm{eV}$ energy transfers, and for $\mathbf{Q}=2.39$ a.u. at 19 and $60 \mathrm{eV}$. The glitch positions can be calculated easily when the sample geometry is known. ${ }^{38}$ The observed glitches were identified as Bragg occurrences from [313] and [133] for $\mathbf{Q}=2.12$ a.u., and [404] and [044] for $\mathbf{Q}=2.39$ a.u., respectively.

In Fig. 1 we compare the measurements along the [111] and the [100] directions. In some cases, in order to reduce the statistical noise in the figures, $2-4$ adjacent data points were binned together. The error bars show the uncertainty of the data due to counting statistics. They are only shown in the last panel of Fig. 1 and in the first panel of Fig. 2, in all other cases they are too small for representation on the scale shown in the figures. In the following data analysis the original unbinned data were used.

More measurements for intermediate $\mathbf{Q}$ have been carried out in the [111] direction in order to probe the sharp feature at around $17 \mathrm{eV}$ which has been related to plasmon Fano resonances. ${ }^{29,30}$ In general, and except for the $\mathbf{Q}=0.53$ a.u., the dynamic structure factor for $\mathbf{Q}$ in direction [111] shows more fine structure than that for $\mathbf{Q}$ in the [100] direction, which has already been mentioned by Schülke, who related this behavior to the plasmon Fano resonance. ${ }^{29}$

In Fig. 2 we compare the results with available measurements of Schülke et al. ${ }^{29}$ Owing to the recent experimental progress, the resolution of the fine structure of the curves has improved. This is particularly clear in the measurement of $\mathbf{Q}=0.80$ a.u. along [111] for which the kink at around $15 \mathrm{eV}$ is now clearly visible. Moreover, the peak position of the measurement shown in Fig. 2(a) differs from the older measurement of Ref. 29 by about $1 \mathrm{eV}$. The comparison with older theoretical results is discussed below in Sec. V. 


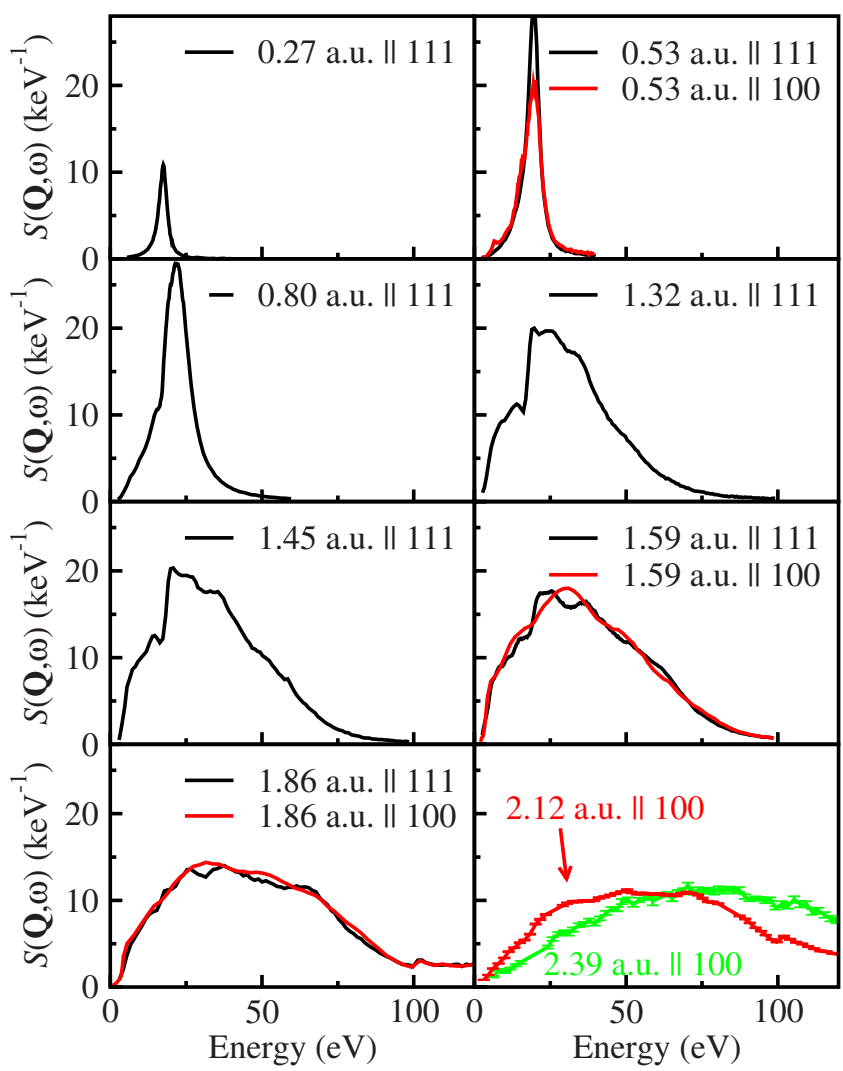

FIG. 1. (Color online) Survey of the measurements along the [111] and the [100] directions, momentum transfers as indicated. Note that all the panels have the same scale.

\section{CALCULATIONS}

\section{A. Approximations of TDDFT}

Most of the calculations have been carried out using linear-response TDDFT on several levels of approximation. The linear-response polarizability $\chi$ and, hence, the inverse dielectric matrix $\varepsilon^{-1}=1+v \chi$, are obtained from the Dysontype screening equation ${ }^{6}$

$$
\chi=\chi^{0}+\chi^{0}\left(v+f_{\mathrm{xc}}\right) \chi,
$$

where $\chi^{0}$ is the independent-particle polarizability, $v$ the bare Coulomb interaction, and the kernel $f_{\mathrm{xc}}$ the functional derivative of the Kohn-Sham exchange-correlation potential $v_{\mathrm{xc}}$ with respect to the electron density. $\chi^{0}$ is given by the following expression in reciprocal space

$$
\begin{aligned}
\chi_{\mathbf{G}_{\mathbf{G}} \mathbf{G}^{\prime}}^{0}(\mathbf{q}, \omega)= & \frac{2}{\Omega} \sum_{\mathbf{k}_{i}} \sum_{i, j}\left(f_{\mathbf{k}_{j} j}-f_{\mathbf{k}_{i}}\right) \\
& \times \frac{\left\langle\mathbf{k}_{j} j\left|e^{-i(\mathbf{q}+\mathbf{G}) \mathbf{r}}\right| \mathbf{k}_{i} i\right\rangle\left\langle\mathbf{k}_{i} i\left|e^{i\left(\mathbf{q}+\mathbf{G}^{\prime}\right) \mathbf{r}^{\prime}}\right| \mathbf{k}_{j} j\right\rangle}{\omega-\left(\epsilon_{\mathbf{k}_{i} i}-\epsilon_{\mathbf{k}_{j} j}\right)+i \eta}
\end{aligned}
$$

in which the solutions of the (static) Kohn-Sham equation are used for the energies (band structure) and for the (Bloch) wave functions. The response function is normalized with the volume $\Omega=\Omega_{\text {cell }} N_{\mathbf{k}}$, i.e., the volume of the cell times the number of $\mathbf{k}$ points. The $f_{\mathbf{k}_{i} i}$ and $f_{\mathbf{k}_{j} j}$ are the respective occupation numbers. Both the indices $i$ and $j$ span the whole
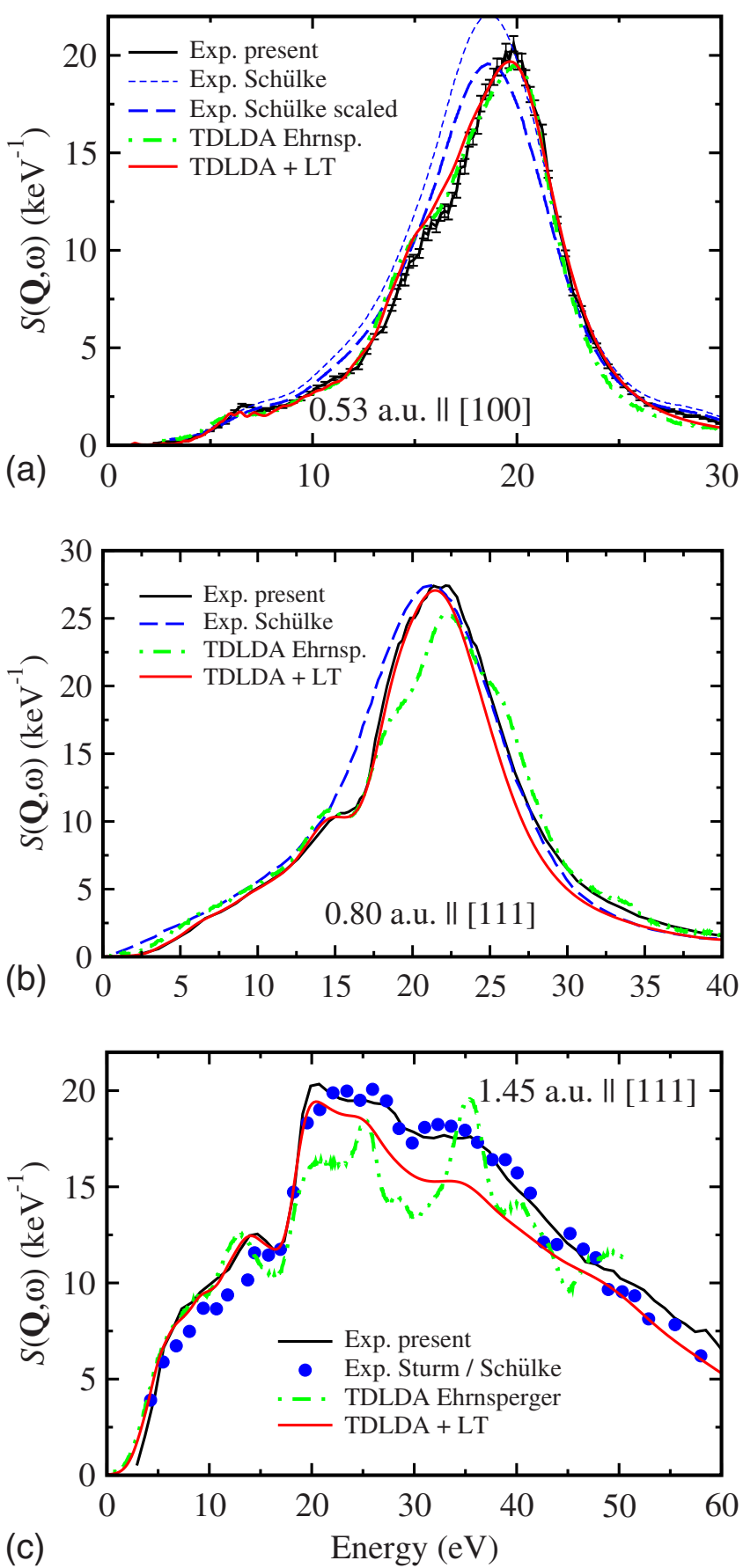

FIG. 2. (Color online) Comparison of the measured and calculated spectra with available literature: measurements of Schülke et al. (Ref. 29) and Sturm et al. (Ref. 39) as well as TDLDA calculations of Ehrnsperger and Bross (Ref. 22) are compared with the present IXS result as well as with the present result of TDLDA including lifetimes as described in Sec. IV B. The result of Schülke et al. for $\mathbf{Q}=0.53$ a.u. along [111] has been rescaled in order to appear with the same peak height as our measured result.

range of all valence and all conduction bands. The expression contains, therefore, the resonant $\left(\epsilon_{\mathbf{k}_{i} i}-\epsilon_{\mathbf{k}_{j} j}>0\right.$, i.e., $i=c, j=v)$ and the antiresonant $\left(\epsilon_{\mathbf{k}_{i} i}-\epsilon_{\mathbf{k}_{j} j}<0\right.$, i.e., $i=v$, $j=c$ ) contribution, depending on the occupation numbers $f_{\mathbf{k}_{i} i}$ and $f_{\mathbf{k}_{j}}, i, j \in\{c, v\}$. Due to conservation of crystal momen- 
tum, the wave vector $\mathbf{k}_{j}$ is restricted according to $\mathbf{k}_{j}=\mathbf{k}_{i}+\mathbf{q}$.

All quantities, $\chi, \chi^{0}, f_{\mathrm{xc}}$, and $\varepsilon$ are functions of frequency $\omega$ and vector $\mathbf{q}$ in the Brillouin zone (BZ). Moreover, they are matrices in the reciprocal-lattice vectors $\mathbf{G}$. If $\mathbf{Q}$ lies outside the $\mathrm{BZ}, \chi_{\mathbf{G}, \mathbf{G}^{\prime}}^{0}(\mathbf{Q}, \omega)=\chi_{\mathbf{G}+\tilde{\mathbf{G}}, \mathbf{G}^{\prime}+\tilde{\mathbf{G}}}^{0}(\mathbf{q}, \omega)$ with $\mathbf{Q}=\mathbf{q}$ $+\widetilde{\mathbf{G}}$. Crystal local-field effects arise from the off-diagonal elements in the inversion. Without them, the matrix ${ }^{40}$ is diagonal and $\varepsilon_{M}(\mathbf{Q}, \omega)=\varepsilon(\mathbf{q}, \omega)_{\mathbf{G}, \mathbf{G}}$ with $\mathbf{Q}=\mathbf{q}+\mathbf{G}$.

In the present work, we use the following approximations:

(i) RPA, in which $\chi^{0}$ is constructed with the Kohn-Sham band structure and wave functions, and $f_{\mathrm{xc}}=0$. This includes crystal local-field effects via the Coulomb potential but neglects the exchange and correlation contributions.

(ii) $G W$-RPA: same approach, but with band structure corrected for self-energy effects within the $G W$ approximation. ${ }^{1}$ This means that the excitation is described on the level of single excited quasiparticles (QPs), whereas the electronhole interaction is neglected.

(iii) Adiabatic local-density approximation ALDA/ TDLDA: using $\chi^{0}$ as in (i) but the local, static approximation of the kernel,

$$
f_{\mathrm{xc}}\left(\mathbf{r}, \mathbf{r}^{\prime}\right)=\delta\left(\mathbf{r}-\mathbf{r}^{\prime}\right)\left\{d v_{\mathrm{xc}}^{\mathrm{LDA}}(\rho(\mathbf{r})) / d \rho(\mathbf{r})\right\} .
$$

(iv) long-range contribution approximation (LRC) (Refs. 7, 15, and 16): $\chi^{0}$ as in (ii), i.e., with $G W$-corrected energies but $f_{\mathrm{xc}}\left(\mathbf{Q}, \mathbf{G}=\mathbf{G}^{\prime}=0, \omega\right)=\left[f_{\mathrm{xc}}(\mathbf{q}, \omega)\right]_{\mathbf{G G}}=-\left(\alpha+\beta \omega^{2}\right) / Q^{2}$. This simulates an $f_{\mathrm{xc}}$ derived from many-body perturbation theory ${ }^{7}$ as an approximation. In particular, it shows the asymptotic $1 / r$ behavior of the kernel in real space. We use the parameters $\alpha$ and $\beta$ determined for silicon at vanishing momentum transfer. ${ }^{16}$

\section{B. Broadening and lifetime effects}

In addition to the customary broadening applied by convoluting the spectra with a Gaussian in order to represent the energy resolution of the experiment, we introduce an energydependent lifetime (LT) broadening, following the idea of Mukhopadhyay et al., ${ }^{41}$ as discussed by Rahman and Vignale. ${ }^{42}$ This is done by replacing the imaginary $i \eta$ in Eq. (7) by the sum of the imaginary energies corresponding to the lifetimes of the electrons and holes, and using the modified independent-particle polarizability

$$
\begin{aligned}
\chi_{\mathbf{G}, \mathbf{G}^{\prime}}^{0, \mathrm{LT}}(\mathbf{q}, \omega)= & \frac{2}{\Omega} \sum_{\mathbf{k}_{i}} \sum_{i, j}\left(f_{\mathbf{k}_{j} j}-f_{\mathbf{k}_{i} i}\right) \\
& \times \frac{\left\langle\mathbf{k}_{j} j\left|e^{-i(\mathbf{q}+\mathbf{G}) \mathbf{r}}\right| \mathbf{k}_{i} i\right\rangle\left\langle\mathbf{k}_{i} i\left|e^{i\left(\mathbf{q}+\mathbf{G}^{\prime}\right) \mathbf{r}^{\prime}}\right| \mathbf{k}_{j} j\right\rangle}{\omega-\left(\epsilon_{\mathbf{k}_{i} i}-\epsilon_{\mathbf{k}_{j} j}\right)+i\left|\Gamma_{\mathbf{k}_{i} i}\right|+i\left|\Gamma_{\mathbf{k}_{j} j}\right|}
\end{aligned}
$$

in Eq. (6). This corresponds to the assumption that electrons and holes decay with a lifetime $\propto 1 / \Gamma_{\mathbf{k}_{i} i}$. The respective imaginary energies are taken from a fit of the modulus of the imaginary part of the self-energy matrix elements of Fleszar and Hanke, Ref. 43, which are very similar to the results of Arnaud et $a l .{ }^{44}$ The underlying assumptions are that (i) TDLDA does essentially not describe lifetime broadening and (ii) the main effect beyond TDLDA stems from the finite lifetimes of the electrons and the holes individually, without lifetime corrections from the electron-hole interaction. The same applies to the RPA, the $G W$-RPA, and the LRC calculations.

While the customary use of a finite $\eta$ in the calculation of $\chi^{0}$ produces an energy-independent broadening, we are, with the present procedure, including approximately the broadening corresponding to each transition. The sign of the added energies in Eq. (8) is such that the causality remains unchanged for the retarded $\chi^{0}$ which we have to calculate. The inclusion of lifetime effects in a similar way has already been done by Schülke et al. ${ }^{29}$ who found that this improved their free-electron results.

A second and simpler form of introducing an energydependent broadening, called in the following "postprocessing," has also been tested. In this case, the spectra $f(\omega)$ obtained using a very small broadening parameter $\eta$ in the calculation of $\chi_{0}$ in Eq. (7) were postprocessed by convoluting them with a Lorentzian of energy-dependent width according to

$$
f_{\text {postproc }}(\omega)=\frac{1}{\pi} \int d \omega^{\prime} \frac{\eta(\omega)}{\left(\omega-\omega^{\prime}\right)^{2}+\eta^{2}(\omega)} f\left(\omega^{\prime}\right) .
$$

The energy dependence of $\eta(\omega)$ has been chosen in the same way as before from the self-energy matrix elements of Ref. 43. The result of this inclusion of the lifetimes is shown in Figs. 3 and 4 for the TDLDA results. While the effects are small for the smallest measured $\mathbf{Q}$, they become important for larger $\mathbf{Q}$. The calculation without inclusion of lifetimes shows strongly overestimated peaks which do not appear to this degree in the experiment. These peaks are due to bandstructure effects, as they appear also in the RPA results (not shown). The postprocessing improves the situation but fails to reach agreement with experiment. Moreover, for energies below $25 \mathrm{eV}$ the spectra appear washed out and overbroadened. This is most clearly seen in the curve for $\mathbf{Q}$ $=1.32$ a.u. in the [111] direction, cf. Fig. 3 .

By contrast, the approximate inclusion of the lifetimes according to Eq. (8) leaves the sharply structured low-energy side intact while reducing the spurious peaks as needed, thus providing evidence that these structures are physical but smoothed out by lifetime effects. The agreement of the TDLDA+LT calculations with experiment, including subtle features at low energy, is excellent. The remaining discrepancy concerns an overestimation of the broadening for higher energies. It becomes apparent that there is a overbroadening in the energy range between roughly 40 and 90 $\mathrm{eV}$, resulting in a shift of oscillator strength to higher energies. Comparison with the result without inclusion of the lifetimes suggests that this is at least partially due to shortcomings of this inclusion of the lifetimes. The resulting underestimate of $S(\mathbf{Q}, \omega)$ for higher energies is found to be stronger for $\mathbf{Q}$ in the [100] direction than for those in the [111] direction.

These shortcomings might be due to the simple fit of the values of Ref. 43 which does not take into account the precise values for each band, nor any directional dependence of the self-energy. However, the anisotropy of the self-energy has been found to be small. ${ }^{45}$ Future $G W$ calculations will 

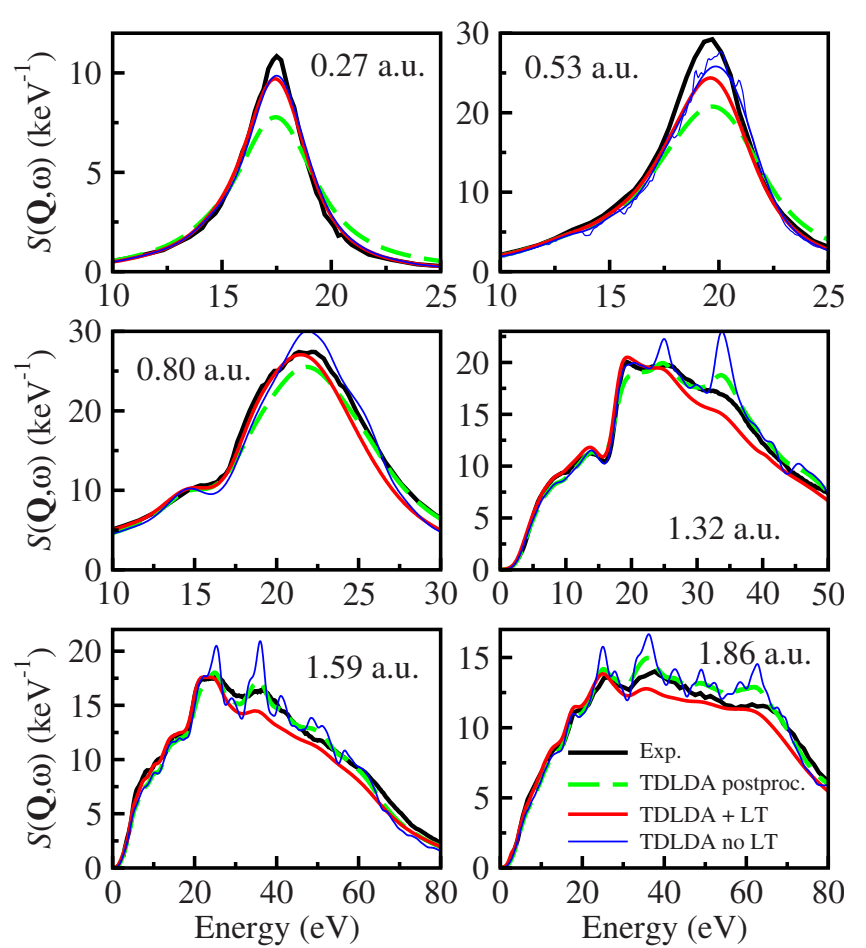

FIG. 3. (Color online) TDLDA results for $\mathbf{Q}$ along [111] using different ways of including lifetime effects, compared with experiment. "TDLDA no LT" stands for a standard TDLDA calculation with a very small constant broadening ( $\eta$ of $0.1 \mathrm{eV}$ ) in the calculation of $\chi^{0}$ according to Eq. (7), but convoluted, like all spectra, with a Gaussian to reflect the energy resolution of the experiment. "TDLDA postproc" is the result of a convolution of the spectrum with an energy-dependent Lorentzian. "TDLDA+LT" reports the result of the inclusion of lifetimes described in Sec. IV B.

answer the question by associating every state with its proper self-energy matrix elements. Another source of the discrepancy might be the change in the lifetimes with respect to the single-QP lifetimes due to electron-hole interaction. This effect is obviously neglected in our calculation. Moreover, Fleszar and Hanke show a substantial difference of the selfenergy matrix elements, in particular for higher energy, depending on whether or not a vertex correction has been used
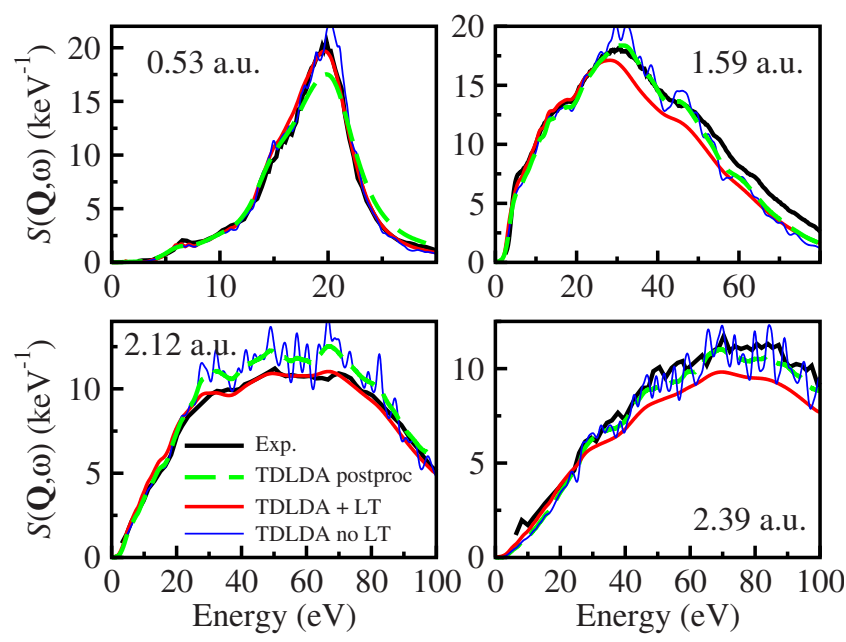

FIG. 4. (Color online) Same as Fig. 3 for $\mathbf{Q}$ along [100]. in the $G W$ calculations. ${ }^{43}$ Very strong effects of vertex corrections on the lifetimes have also been found for $\mathrm{LiF}^{46} \mathrm{Con}$ clusive tests appear to be unavailable at present. Improvements concerning the calculation and the inclusion of the lifetimes must therefore be left to future calculations. However, for our conclusions with respect to the role played by the lifetimes, the present results are sufficient.

\section{Lifetime effects in the exchange-correlation kernel}

The inclusion of the lifetime effects introduced via the $\chi^{0, \mathrm{LT}}$ is not rigorous since it is the true Kohn-Sham independent-particle polarizability $\chi^{0}$ which appears in Eq. (6). However, in the independent-particle approximation, the electrons and holes have infinite lifetimes. Therefore, we are looking for an approximation of $f_{\mathrm{xc}}$ that includes the lifetime effects. To start with, we tested the Gross-Kohn kernel ${ }^{6}$ because it does have an energy-dependent imaginary part. However, this changed only very little in our results compared to the TDLDA (not shown).

On the other hand, the polarizability $\chi$ calculated using Eq. (6) but with the modified $\chi^{0, \mathrm{LT}}$ yields an excellent result in agreement with experiment. Therefore we search a kernel $f_{\mathrm{xc}}$ which obtains equivalent results using Eq. (6) with the original $\chi^{0}$. This is done by requiring that the polarizability obtained by the modified Eq. (6),

$$
\chi=\chi^{0, \mathrm{LT}}+\chi^{0, \mathrm{LT}}\left(v+f_{\mathrm{xc}}^{\mathrm{ALDA}}\right) \chi
$$

be equal to the one obtained with the original Eq. (6) and the true independent-particle polarizability $\chi^{0}$, but now with an $f_{\mathrm{xc}}^{\mathrm{LT}}$ that represents the lifetime effects

$$
\chi=\chi^{0}+\chi^{0}\left(v+f_{\mathrm{xc}}^{\mathrm{ALDA}}+f_{\mathrm{xc}}^{\mathrm{LT}}\right) \chi .
$$

Formally combining Eqs. (10) and (11) yields the desired approximation of the kernel as

$$
f_{\mathrm{xc}}^{\mathrm{LT}}=\chi_{0}^{-1}-\chi_{0, \mathrm{LT}}^{-1} .
$$

In Fig. 5 we show the resulting $f_{\mathrm{xc}}^{\mathrm{LT}}$ for different $\mathbf{Q}$. Note that, like $\varepsilon^{-1}(\mathbf{q}, \omega)_{\mathbf{G}, \mathbf{G}^{\prime}}$, all the quantities discussed here, $f_{\mathrm{xc}}, \chi_{0}$, and $\chi_{0}^{-1}$, are matrices in $\mathbf{G}$, with $\mathbf{q}$ in the Brillouin zone.

It is interesting to note that the kernel is both energy and $\mathbf{Q}$ dependent. The lifetime description in this way introduces an imaginary part in the kernel and modifies the real part, as compared to the ALDA kernel. The real part of $f_{\mathrm{xc}}^{\mathrm{LT}}$ is indeed important. Neglecting this part in Eq. (11) leads to spectra which deviate appreciably from the result including the lifetimes (not shown). The $\mathbf{Q}$ dependence reflects the nonlocality of the kernel in real space, the energy dependence nonlocality in time. The latter dependence was of course to be expected for the description of lifetime effects, as was the presence of an imaginary part. By contrast, the ALDA kernel is real, local in space, and time independent.

We mention that $\chi^{0}$ is, strictly mathematically, not invertible. ${ }^{49}$ However, inversion using similar matrix sizes as used in the calculation of the spectra, is possible. The result is shown in Fig. 6 . The quantities $\chi_{0, \mathrm{LT}}^{-1}$ and $\chi_{0}^{-1}$ are relatively unstable with respect to the matrix size, i.e., the number of $\mathbf{G}$ vectors. However, this dependence on the number of $\mathbf{G}$ vectors cancels out when the difference of Eq. (12) is taken. The 

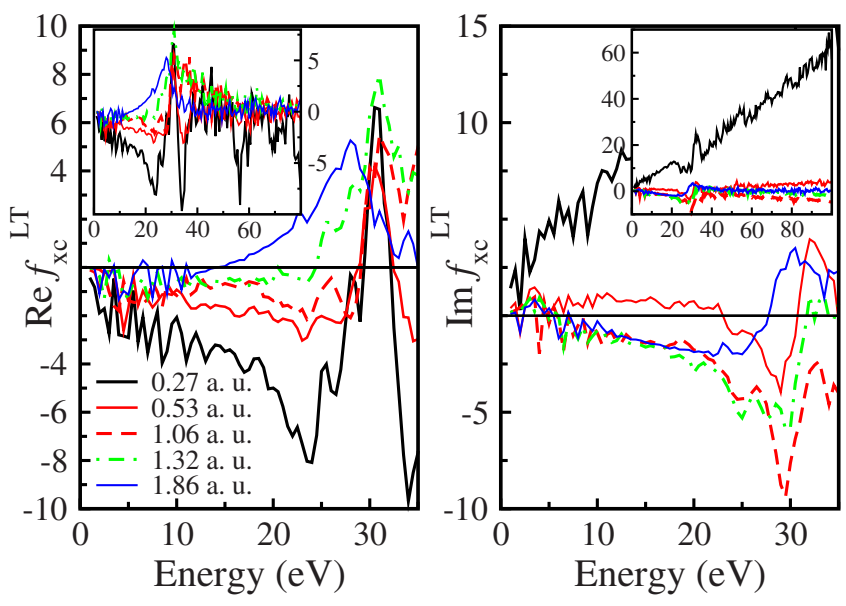

FIG. 5. (Color online) Exchange-correlation kernel $f_{\mathrm{xc}}^{\mathrm{LT}}$ including lifetime effects obtained according to Eq. (12) and using the polarizability obtained with the ALDA kernel and the modified $\chi^{0, \mathrm{LT}}$ of Eq. (8) for the momentum transfers $\mathbf{Q}$ as indicated along [111]. Shown is the diagonal element $\left[f_{\mathrm{xc}}^{\mathrm{LT}}(\mathbf{q}, \omega)\right]_{\mathbf{G}, \mathbf{G}}$, again with $\mathbf{Q}=\mathbf{q}+\mathbf{G}$. The insets show the same graphs on a larger scale.

kernel itself is much more stable than the $\chi_{0, \mathrm{LT}}^{-1}$ and $\chi_{0}^{-1}$ alone which are shown in Fig. 7 for different momentum transfers. The spectra produced with the kernels resulting for different numbers of $\mathbf{G}$ vectors are virtually indistinguishable (not shown). This very limited influence of details such as the matrix size of the kernel is similar to findings reported by Sottile et al. ${ }^{8,47,48}$ and Marini et al. ${ }^{10}$ Finally, we note that, in particular, the behavior of $f_{\mathrm{xc}}^{\mathrm{LT}}$ at high energies should not be overinterpreted because the fit used to represent the imaginary part of the self-energy matrix elements as discussed above is probably only of limited value in this region. Already the spectra calculated with the lifetimes are less good in this region due to an apparent overbroadening that leads to an underestimation of the spectra as discussed in Sec. IV B.

\section{Test of sum rules}

In order to test the numerical approach, we considered the sum rule Eq. (5) for our calculations. We used a calculation
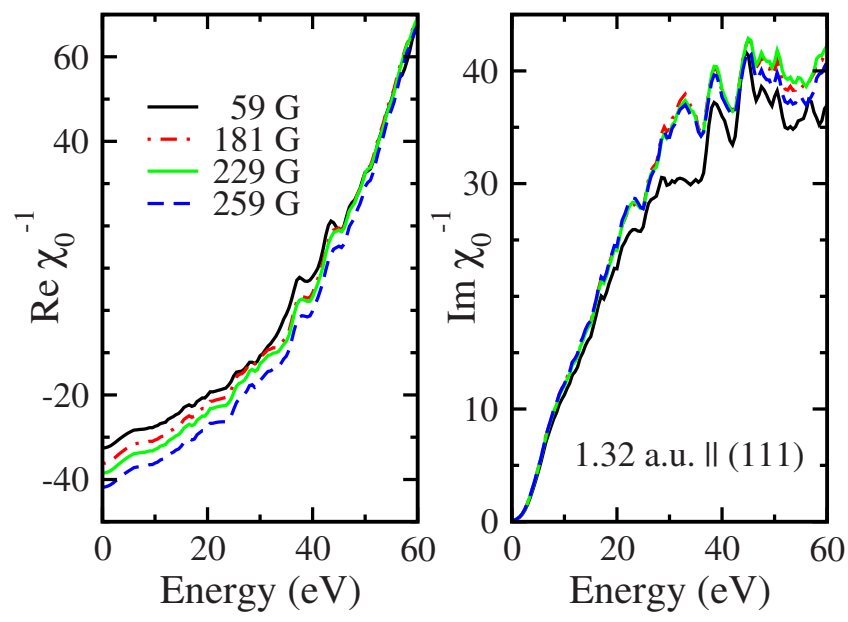

FIG. 6. (Color online) Inversion of the independent-particle polarizability $\chi_{0}^{-1}$ for different numbers of $\mathbf{G}$ vectors. Shown is the diagonal element of the matrix, $\left[\chi_{0}^{-1}(\mathbf{q}, \omega)\right]_{\mathbf{G}, \mathbf{G}}$.
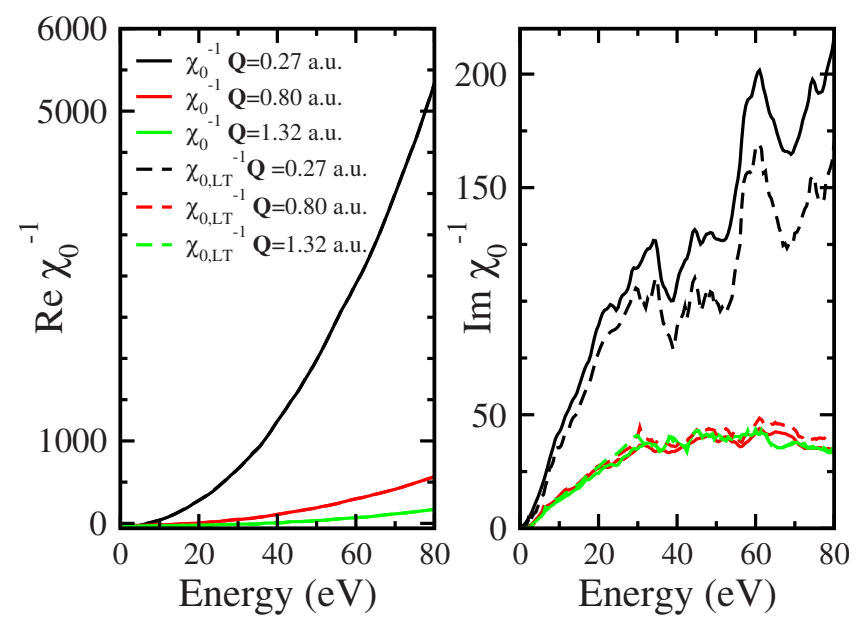

FIG. 7. (Color online) Inverse of the independent-particle polarizability with $\left(\chi_{0, \mathrm{LT}}^{-1}\right)$ and without lifetimes $\left(\chi_{0}^{-1}\right)$ for different momentum transfers. The real parts are almost exactly superimposed on the present scale, i.e., the difference between the two polarizabilities is very small compared to the polarizability itself.

for $\mathbf{Q}=0.27$ a.u. in the [111] direction with 256 shifted $\mathbf{k}$ points, 200 bands, 259 plane waves for the expansion of the wave functions, and $89 \mathbf{G}$ vectors for the dielectric matrix. The calculations were done up to $150 \mathrm{eV}$. An exponential tail was fitted to the curve starting from $75 \mathrm{eV}$. The integral calculated for both TDLDA and RPA was 2.3\% smaller than the exact value. The contribution of the fitted tail was about $1 \%$ of the value of the integral.

The modulus of this deviation of $-2.3 \%$ is of the same order of magnitude as the deviation found by Ehrnsperger and $\mathrm{Bross}^{22}$ for a TDLDA calculation at a similar momentum transfer, which was $1.4 \%$ (i.e., opposite in sign). As mentioned by Ehrnsperger and Bross, ${ }^{22}$ the deviation is apparently caused by small deviations at high energies which are enhanced by the factor of $\omega$ in the sum rule. This applies, in particular, to the fitted tail.

For that reason, a test with the screening sum rule

$$
\operatorname{Re} \varepsilon_{\mathrm{M}}(\mathbf{Q}, \omega=0)=1+\frac{2}{\pi} \int_{0}^{\infty} \frac{d \omega}{\omega} \operatorname{Im} \varepsilon_{\mathrm{M}}(\mathbf{Q}, \omega),
$$

which is a direct consequence of the Kramers-Kronig relations, has been carried out as well. Compared with $\varepsilon_{\mathrm{M}}(\mathbf{Q}, \omega=0)$ calculated directly, the integral Eq. (13) over the TDLDA spectrum is $0.13 \%$ smaller. Also this deviation is on the order of magnitude of that of Ehrnsperger and Bross' corresponding calculation. ${ }^{22}$ The error is smaller than for the sum rule Eq. (5) because the factor $1 / \omega$ suppresses small inaccuracies at high energies, contrary to the factor $\omega$ in Eq. (5).

\section{E. Technical details}

The ground-state calculations, as well as the calculations of the $G W$ corrections, were done with the plane-wave pseudopotential code ABINIT (Ref. 50) using the Perdew-Zunger ${ }^{51}$ parameterization of the exchangecorrelation potential. All calculations except those treating 
the silicon $L_{2,3}$ edge discussed in Sec. V F were done using a Hamann pseudopotential ${ }^{52}$ treating the $3 s$ and $3 p$ electrons as valence electrons. The calculations have been converged with respect to all parameters. In particular, the energy cutoff was $E_{\text {cut }}=16$ hartree. Subsequently, employing the DP code, ${ }^{53} \chi^{0}$ is constructed using the Kohn-Sham wave functions and energies calculated at a regular grid of 2048 off-symmetry ${ }^{54} \mathbf{k}$ points.

Subsequently, Eq. (6) is solved. Depending on the energy range, between 70 and 200 bands, 89 and $259 \mathbf{G}$ vectors for the wave functions, and between 51 and 89 for the matrix have been used. The latter number gives the rank of the matrix for the inversion, which is important for the description of the crystal local-field effects. The parameters have been chosen such that on the scale of the figures no visible change was produced when they were further increased. The $G W$ energy corrections have been calculated with a non-selfconsistent $G_{0} W_{0}$ calculation for ten $\mathbf{k}$ points and then interpolated. All curves have been convoluted with a Gaussian of $1.1 \mathrm{eV}$ FWHM corresponding to the experimental energy resolution, except for $\mathbf{Q}=0.53$ a.u. along [100] where the resolution was $0.2 \mathrm{eV}$.

\section{RESULTS}

\section{A. Dynamic structure factor}

We begin our presentation with a comparison with older calculations. In Fig. 2 we compare our results with available TDDFT calculations of Ehrnsperger and Bross who used an augmented-plane-wave method. ${ }^{22}$ Their calculations use different approximations to the kernel $f_{\mathrm{xc}}$ of Eq. (6), one of them being the ALDA. The main difference of their results from ours is due to our inclusion of the lifetimes. Their results stand in relatively good agreement with experiment except for the peaklike structures at higher energies which are similar to those in our results without inclusion of the lifetime effects. We have thus explained the discrepancies which Ehrnsperger and Bross conjectured to be produced by artifacts of the band-structure calculations. ${ }^{22}$ The remaining differences in the spectra are probably due to slight differences in the methods used for the ground-state calculations.

The results of our calculations are shown in Figs. 8 and 9 along with the experimental curves. For all the measured momentum transfers $\mathbf{Q}$, the description of $S(\mathbf{Q}, \omega)$ in TDLDA+LT is excellent. This applies to the peak positions but also to the fine structures, in particular around $17 \mathrm{eV}$, which are clearly visible in the experiment and which have been related to plasmon Fano resonances. ${ }^{29}$ Indeed, the mixing of plasmons is contained in our calculations including local-field effects. The excellent agreement is consistent with other encouraging results, in particular for the response of $s$ and $p$ valence electrons. ${ }^{18-23}$ We observe that even the RPA calculations yield results which are not completely wrong, although they are far from the quality of the TDLDA results. The latter improves strongly upon the RPA due to its inclusion of many-body effects missing in the latter. The main differences to the TDLDA results are a slightly higher energetic position and a relative lowering of the low-energy side of the spectra. Apart from this, all the structures are present
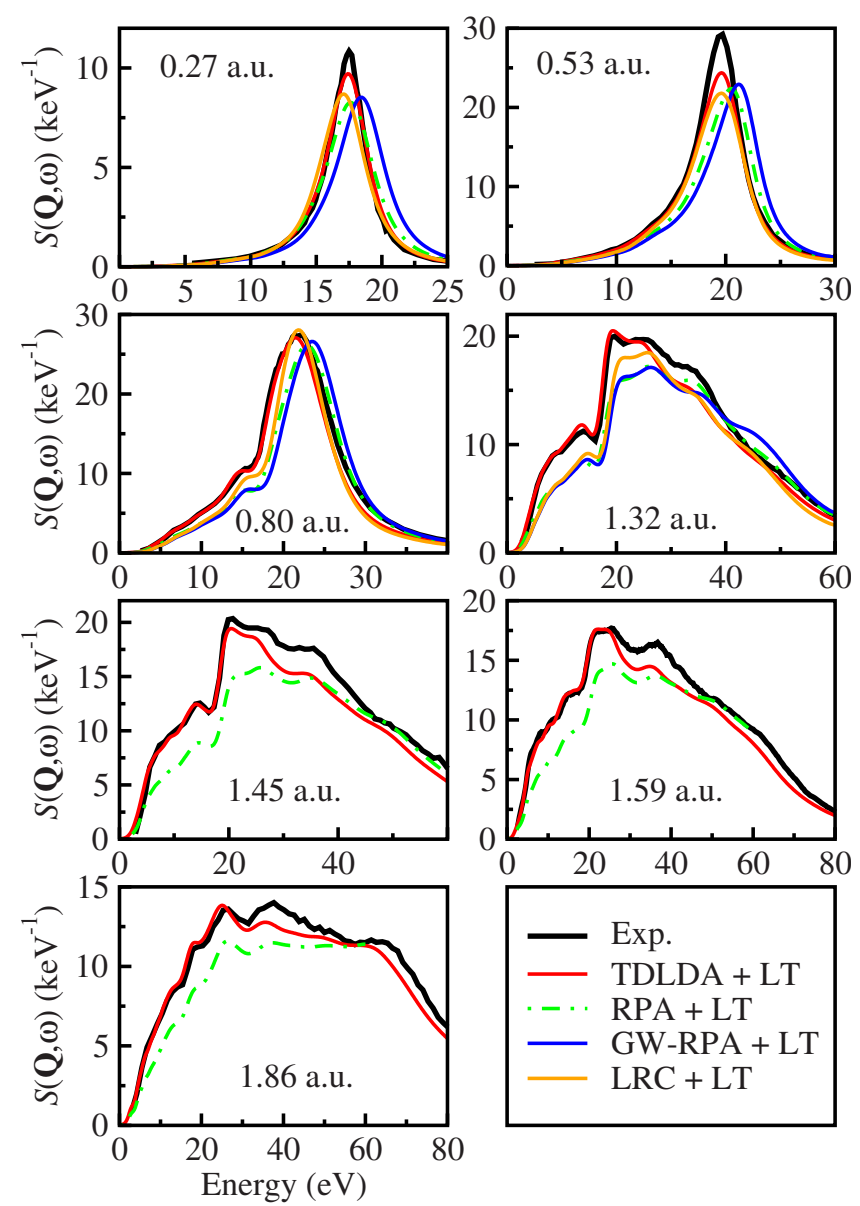

FIG. 8. (Color online) Measured dynamic structure factor $S(\mathbf{Q}, \omega)$ for $\mathbf{Q}$ along [111] compared with calculations using the different approximations.

which means that they can only be due to band-structure effects and crystal local-field effects. The $G W$-RPA calculations, as in the case of optical absorption, shift the RPA result toward higher energies without providing any improvement to the shape of the spectra. This is of course due to the inclusion of the self-energy effects via the $G W$ energies, whereas the electron-hole interaction remains unaccounted for. This picture is consistent for all the measured $\mathbf{Q}$ values, both in the [111] direction and, likewise, in the [100] direction.

The long-range approximation LRC to $f_{\mathrm{xc}}$ produces slightly different effects. Using the parameters $\alpha$ and $\beta$ which for $\mathbf{Q}=0$ yield the correct spectra, ${ }^{16}$ we see that for all the larger $\mathbf{Q}$ it changes the $G W$-RPA curves in the right direction, although not sufficiently to give the correct spectra. This is consistent with the fact illustrated below in Sec. V E that the long-range contribution becomes less and less important for larger momentum transfers. For the smaller $\mathbf{Q}$ values, it fares better. For $\mathbf{Q}=0.53$ a.u. in [111] direction it gives the correct peak position. This might, however, be a fortuitous result. For the smallest $\mathbf{Q}$ measured, the excitonic effects moving the spectra to lower energies are even overestimated, and the peak position is too low.

TDLDA is therefore the method of choice for the calculation of valence spectra at finite momentum transfer in 

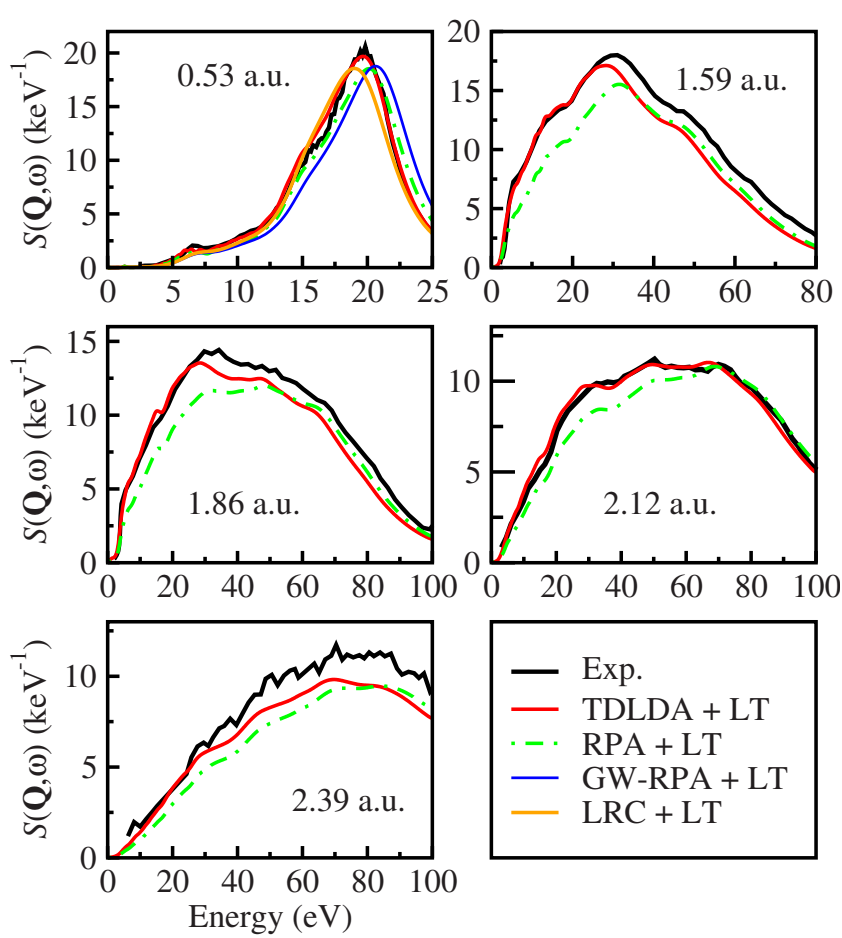

FIG. 9. (Color online) Same as Fig. 8 for momentum transfer along [100].

semiconductors. Besides its quality demonstrated in the present work, its simplicity makes the spectra easily available.

\section{B. Anisotropy}

In order to investigate the description of the anisotropy of the spectra, we show in Fig. 10 the difference spectra obtained by subtracting the spectra in [100] direction from those in [111] direction. Here even more than in the spectra themselves it is evident that the inclusion of the lifetimes is necessary to obtain spectra amenable to clear conclusions. Moreover, the RPA description is already rather good, except for the overestimate of the energetic positions of some features. This shows that the anisotropy is almost exclusively determined by band-structure and crystal local-field effects. This will be discussed below in Sec. V D.

\section{Dielectric function from Kramers-Kronig relations}

While the dynamic structure factor $S(\mathbf{Q}, \omega)$ and the energy-loss function have been studied extensively before, much less is known concerning the dielectric function $\varepsilon_{\mathrm{M}}(\mathbf{Q}, \omega)$ itself. The high quality of the present experiment enabled the extraction of $\varepsilon_{\mathrm{M}}(\mathbf{Q}, \omega)$ from the experimental $S(\mathbf{Q}, \omega)$ using the Kramers-Kronig (KK) relations. This is done by eliminating one of $\operatorname{Re} \varepsilon_{\mathrm{M}}$ or $\operatorname{Im} \varepsilon_{\mathrm{M}}$ in $S(\mathbf{Q}, \omega) \propto$ $-\operatorname{Im} 1 / \varepsilon_{M}=-\operatorname{Im} \varepsilon_{M} /\left[\left(\operatorname{Re} \varepsilon_{M}\right)^{2}+\left(\operatorname{Im} \varepsilon_{M}\right)^{2}\right]$ and thus obtaining the other. In principle, this procedure includes an integration up to infinite energy. Tests showed that the procedure does not depend strongly on the length and the shape of the tail fitted to the experimental spectra at high energies (not

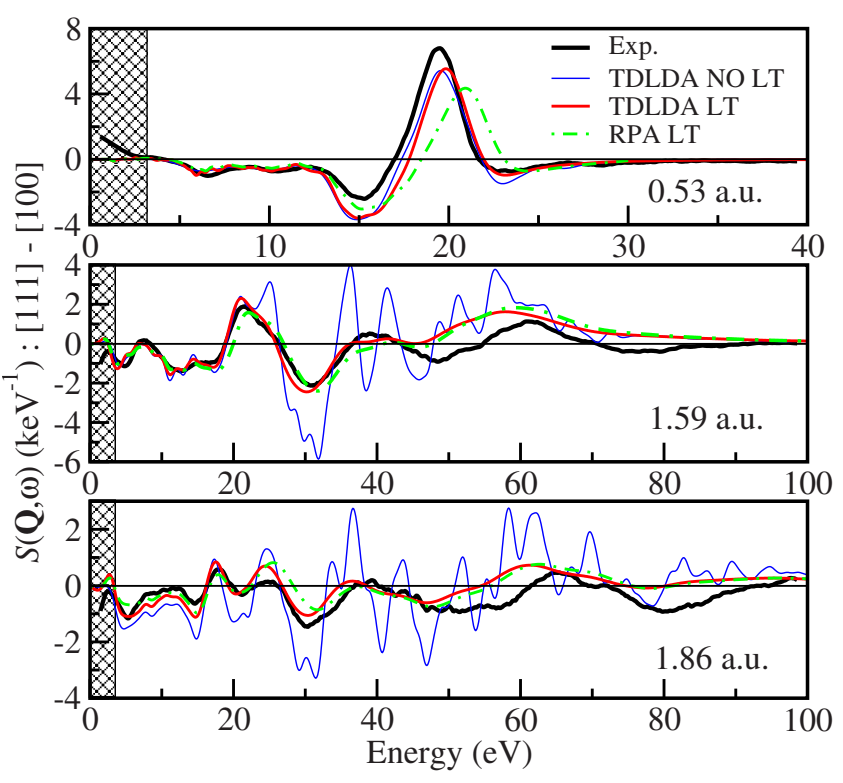

FIG. 10. (Color online) Anisotropy of the dynamic structure factor $S(\mathbf{Q}, \omega)$, i.e., difference $S(\mathbf{Q}, \omega)\|[111]-S(\mathbf{Q}, \omega)\|[100]$. The experimental curves have been smoothed using a "boxcar convolution" after taking the difference. The boxes indicate the area for which the experimental spectra are not reliable due to the extraction of the elastic peak.

shown). However, it proves extremely sensitive to changes in the normalization. This is illustrated in Fig. 11 for two values of $\mathbf{Q}$, one rather small, the other rather large. The figure shows the result of the extraction using the KK relations for the normalized spectra but also for the same spectra with the normalization factor changed by up to $10 \%$. This corresponds roughly to the uncertainty which is inherent in the experimental spectra and in their normalization. For the larger $\mathbf{Q}$, there is some influence, but the result remains accessible within the treated range. By contrast, in the case of

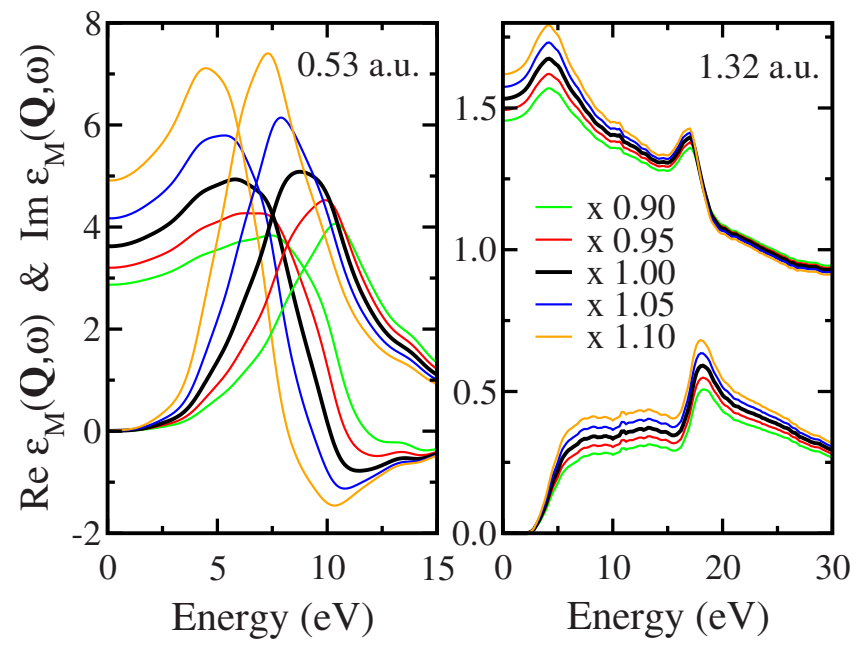

FIG. 11. (Color online) Influence of normalization of $S(\mathbf{Q}, \omega)$ on the extraction of the dielectric function using the Kramers-Kronig relations. Shown is the result starting from a calculation of $S(\mathbf{Q}, \omega)$ unchanged $(\times 1.00)$ or changed by the factors as indicated in the legend. 

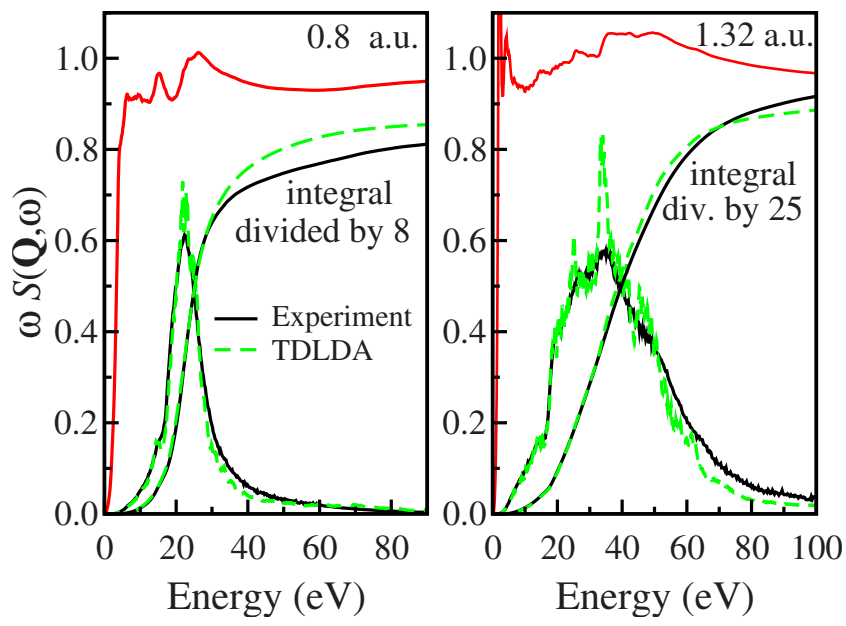

FIG. 12. (Color online) Procedure of normalization of $S(\mathbf{Q}, \omega)$ used for the extraction of the dielectric function from the measured dynamic structure factor using the Kramers-Kronig relations. We show the product $\omega S(\mathbf{Q}, \omega)$ as well as its integral $\int_{0}^{\omega} d \omega^{\prime} \omega^{\prime} S\left(\mathbf{Q}, \omega^{\prime}\right)$ along with the ratio of the two integrals.

the smaller $\mathbf{Q}$, the result changes so strongly that clear conclusions from the comparison with the calculation become impossible. For that reason, we have employed the following procedure. ${ }^{30}$

We calculate, for both the experimental and the TDLDA result, the normalization integral $\int_{0}^{\omega} d \omega^{\prime} \omega^{\prime} S\left(\mathbf{Q}, \omega^{\prime}\right)$ which for $\omega \rightarrow \infty$ yields the sum rule that should also be satisfied in TDLDA. ${ }^{55,56}$ Along with the excellent agreement between the experimental and the TDLDA results, this allows us to assume that at the high-energy tail the value of the integrals should be the same. We take the largest and the smallest ratio of the two within the considered range as maximal correction factors to the normalization. This defines the error bar for $\varepsilon_{\mathrm{M}}(\mathbf{Q}, \omega)$. The procedure is illustrated in Fig. 12 for two values of $\mathbf{Q}$ (see footnote 56 ).

The dielectric function extracted in this way from the experiment is shown in Figs. 13-16. Note that the smallest $\mathbf{Q}$ in [111] direction has been omitted because the procedure discussed above did not yield any reliable result that could have been compared with the calculations.

The results show consistently that, as in the case of the structure factor, also for the dielectric function the agreement between the TDLDA results and experiment is excellent. This stands in clear contrast to the case of $\mathbf{Q}=0$ where the TDLDA does not give reasonable absorption spectra even though it obtains decent agreement for the plasmon peak. The success of the TDLDA is in fact due to exchangecorrelation effects represented in the TDLDA and not just to the classical Hartree contribution; the result of the RPA, and even more of the $G W$-RPA, stand in much poorer agreement with experiment. Since the difference between $G W$-RPA and experiment is mainly due to the electron-hole interaction, we conclude that TDLDA reproduces significant excitonic effects for finite momentum transfer. These effects are only implicitly contained in TDLDA. The full TDDFT kernel contains a contribution that simulates the quasiparticle gap correction as in $G W$-RPA, and a second term responsible for the electron-
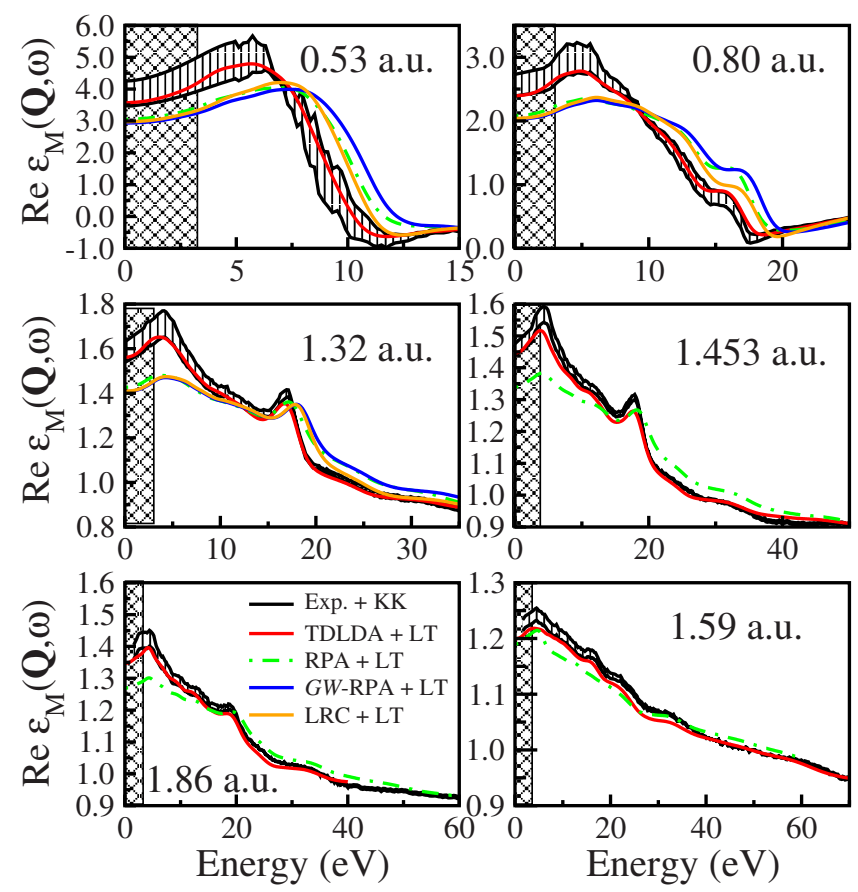

FIG. 13. (Color online) Re $\varepsilon_{\mathrm{M}}(\mathbf{Q}, \omega)$ extracted from the measured $S(\mathbf{Q}, \omega)$ using the Kramer-Kronig relations for momentum transfer along [111]. Colors as in Fig. 8. The boxes cover the energy range for which the procedure does not yield reliable results due to the errors introduced by the extraction of the elastic peak.

hole interaction. ${ }^{11,57}$ The two contributions cancel partially and TDLDA merely has to reproduce the remaining correction. Our results demonstrate that the latter should have a significant short-range contribution that is well described by a local approximation.
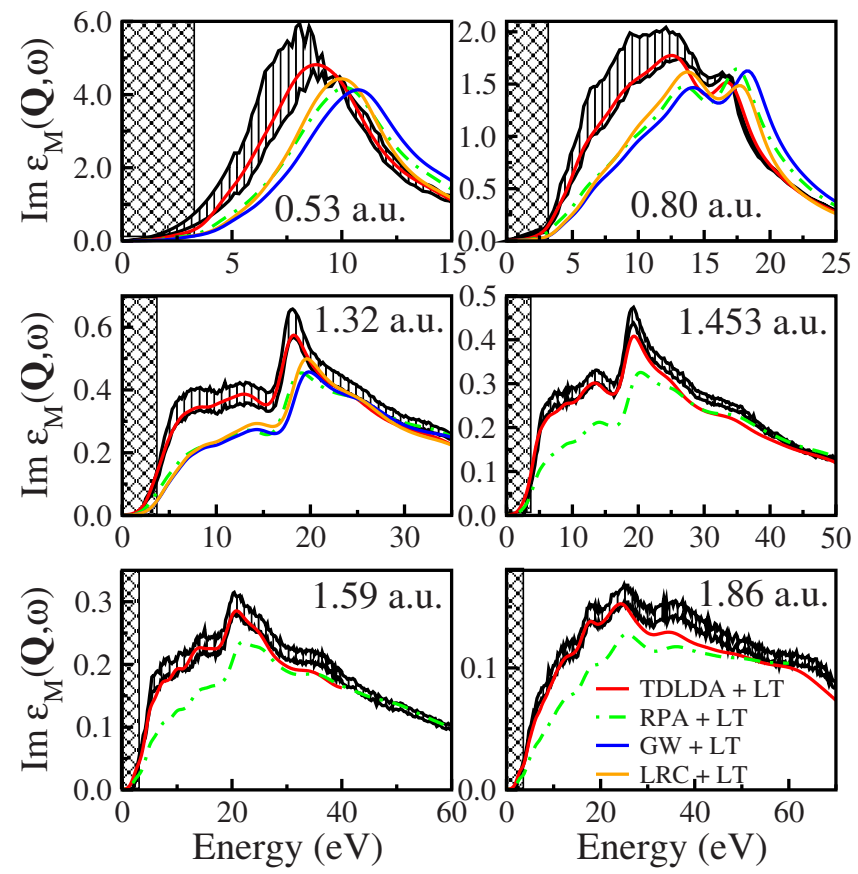

FIG. 14. (Color online) Same as Fig. 13 for the imaginary part $\operatorname{Im} \varepsilon_{\mathrm{M}}(\mathbf{Q}, \omega)(\mathbf{Q}$ along [111]). 

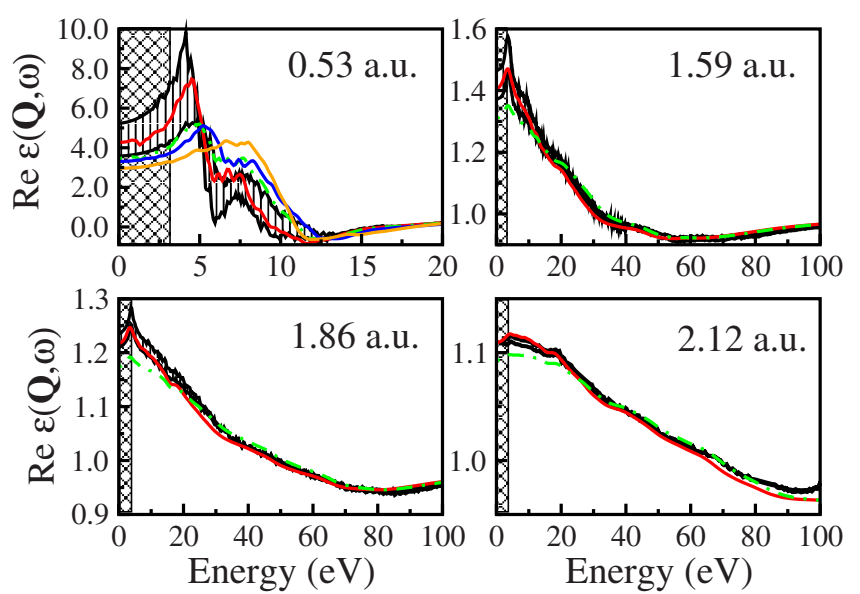

FIG. 15. (Color online) Same as Fig. 13 for $\mathbf{Q}$ along [100].

Figures 13-16 show also the result obtained with the LRC kernel. This approximation corrects the $G W$-RPA, but by far not enough; a weak long-range contribution alone is now not sufficient to explain the spectra. LRC performs better for the plasmon peak (not shown here) but still slightly less well than TDLDA. These findings are systematic and apply to both the imaginary and the real part of the dielectric function.

\section{Crystal local-field effects}

As highlighted in our recent work, ${ }^{30}$ the crystal local-field effects (CLFE) are very important for the present spectra, in particular for the coupling between the resonant and the antiresonant part of the polarizability. In order to obtain a clear representation of the CLFE we show in Figs. 17 and 18 RPA spectra with and without the inclusion of the CLFE. (The spectra shown here have been calculated without the inclusion of lifetime effects.) In practice, the omission of the CLFE is done by neglecting the off-diagonal elements of the dielectric matrix in the inversion. For the RPA this is equivalent to using only the $\mathbf{G}=0$ component of the Fourier transform of the Coulomb potential in Eq. (6), as outlined in Ref. 17.
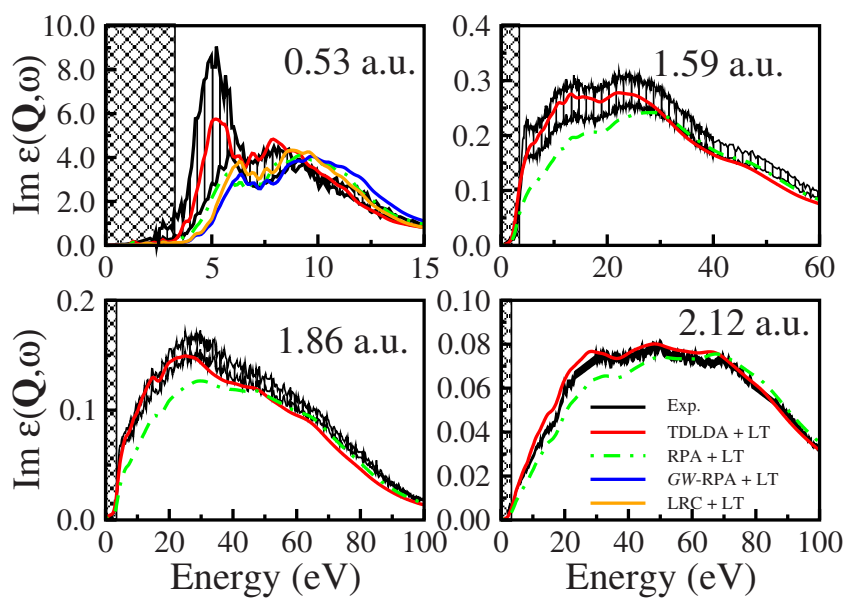

FIG. 16. (Color online) Same as Fig. 14 for $\mathbf{Q}$ along [100].
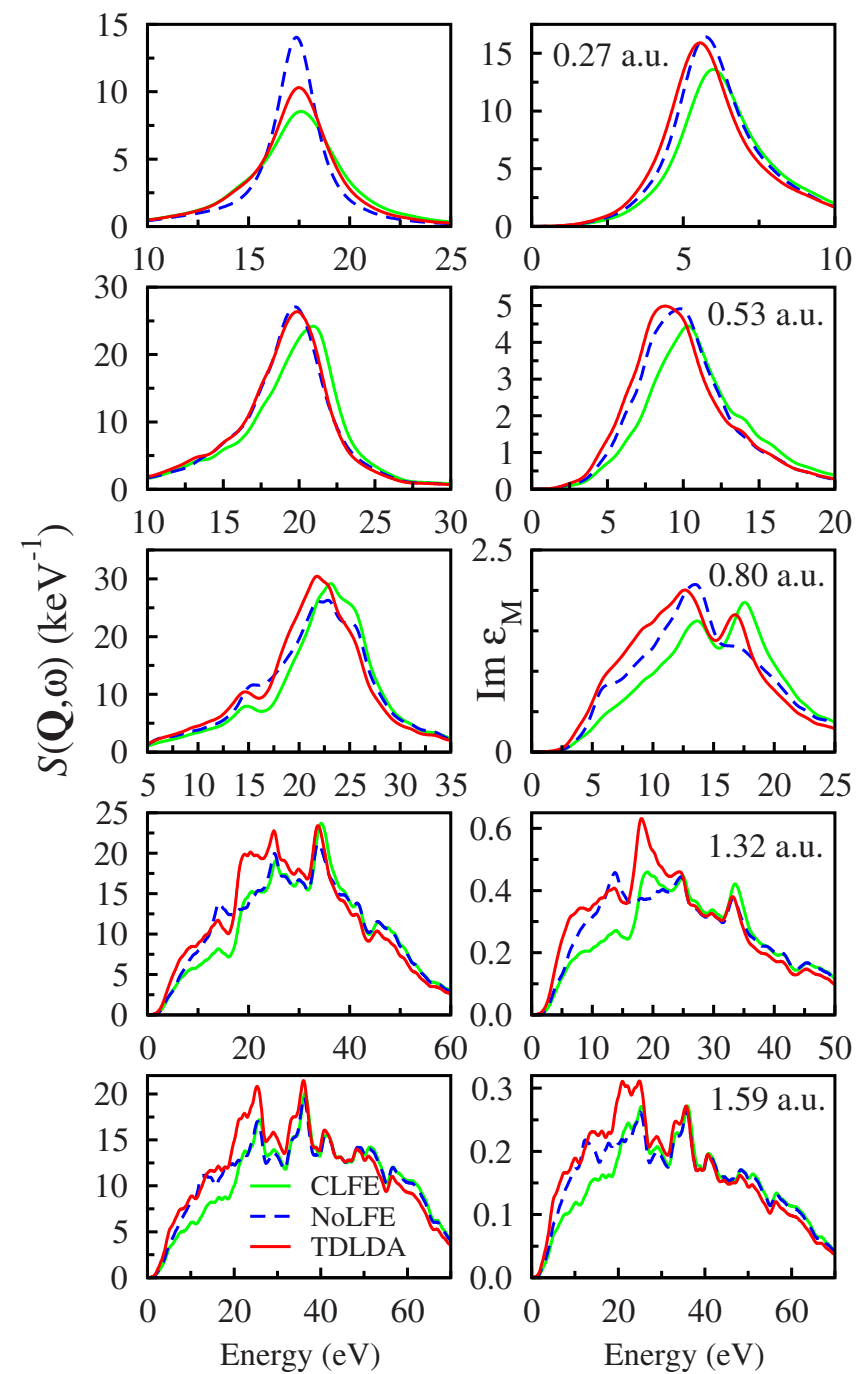

FIG. 17. (Color online) Influence of the crystal local-field effects on the dynamic structure factor (left panels) and on the imaginary part of the dielectric function (right panels) for $\mathbf{Q}$ in the [111] direction. The RPA results with and without CLFE are shown along with the TDLDA. Shown are the results without the inclusion of lifetime effects but broadened with a Gaussian broadening of 1.0 $\mathrm{eV}$.

The CLFE are somewhat more visible in the spectra in [111] direction. Moreover, the main influence is found in the region between 5 and $20 \mathrm{eV}$. On the other hand, the influence of the CLFE on $S(\mathbf{Q}, \omega)$ and on the dielectric function is more or less comparable. We note that the CLFE shift spectral weight to higher energies. This goes in the opposite direction compared to the many-body effects introduced by the ALDA kernel. In some situations (cf., e.g., $\mathbf{Q}=0.53$ and 0.80 a.u. along [111]) the RPA without CLFE is therefore closer to the TDLDA and, therefore, to the experimental result than the full RPA including CLFE.

The CLFE in our results are comparable but larger than those in the results of Montano et al. for 3C-SiC (Ref. 58) which are, however, not as visible in changing a peak position. The effects we find are likewise larger than the CLFE found in diamond by Waidman et al. ${ }^{23}$ Similarly large effects have been found by Gurtubay et al. for $\mathrm{TiO}_{2}{ }^{59}$ 


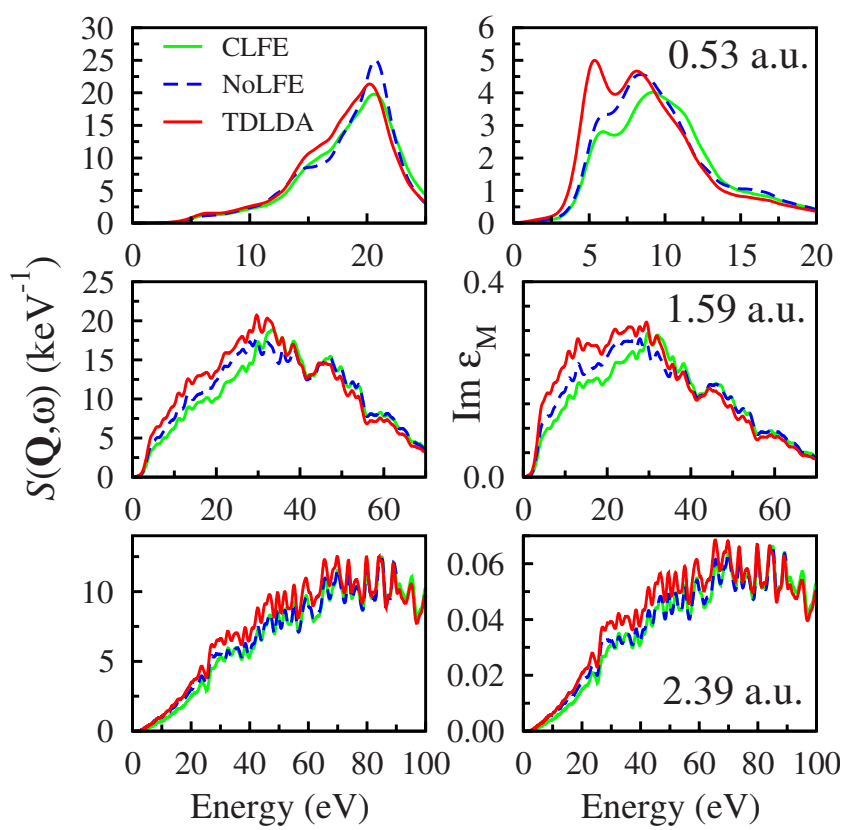

FIG. 18. (Color online) Same as Fig. 17 for $\mathbf{Q}$ along [100].

Finally, we note that the CLFE are responsible for most of the anisotropy in the spectra. This is illustrated in Fig. 19 where we plot the RPA result with and without CLFE in comparison with the experimental result and the TDLDA. As mentioned before, the anisotropy is already well described by the complete RPA calculation. It has been noted already by Montano et al. that the description of the anisotropy requires the inclusion of the CLFE. On the other hand, when the CLFE are neglected, the difference spectra become very different and the region between 0 and $30 \mathrm{eV}$ does not at all represent the anisotropy correctly. This is consistent with the

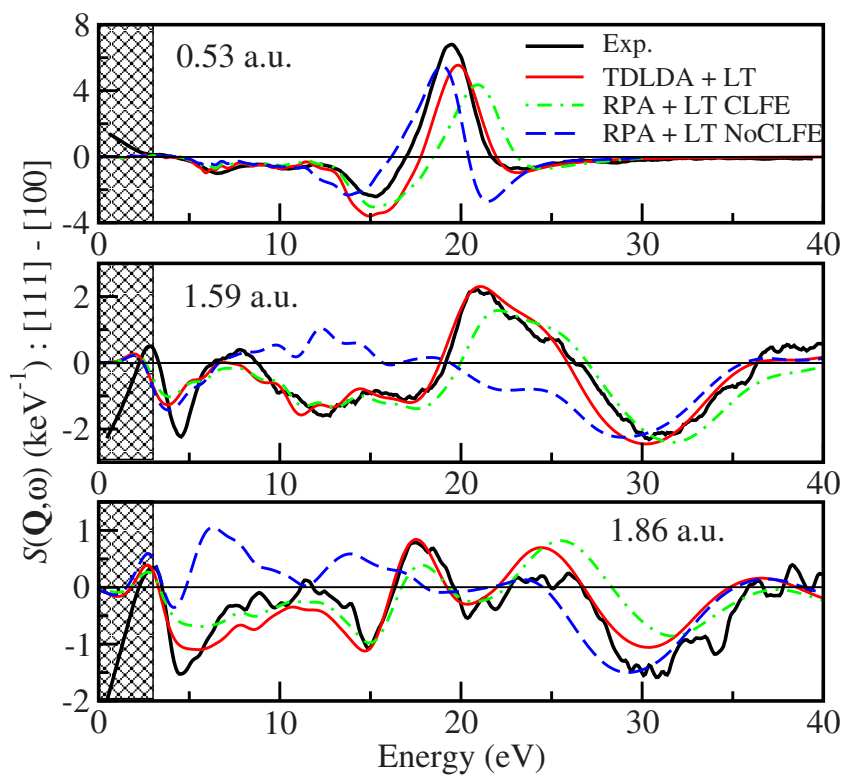

FIG. 19. (Color online) Influence of the crystal local-field effects on the anisotropy of the dynamic structure factor $S(\mathbf{Q}, \omega)$, i.e., on the difference $S(\mathbf{Q}, \omega)\|[111]-S(\mathbf{Q}, \omega)\|[100]$. The experimental curves have been smoothed using a boxcar convolution after taking the difference.

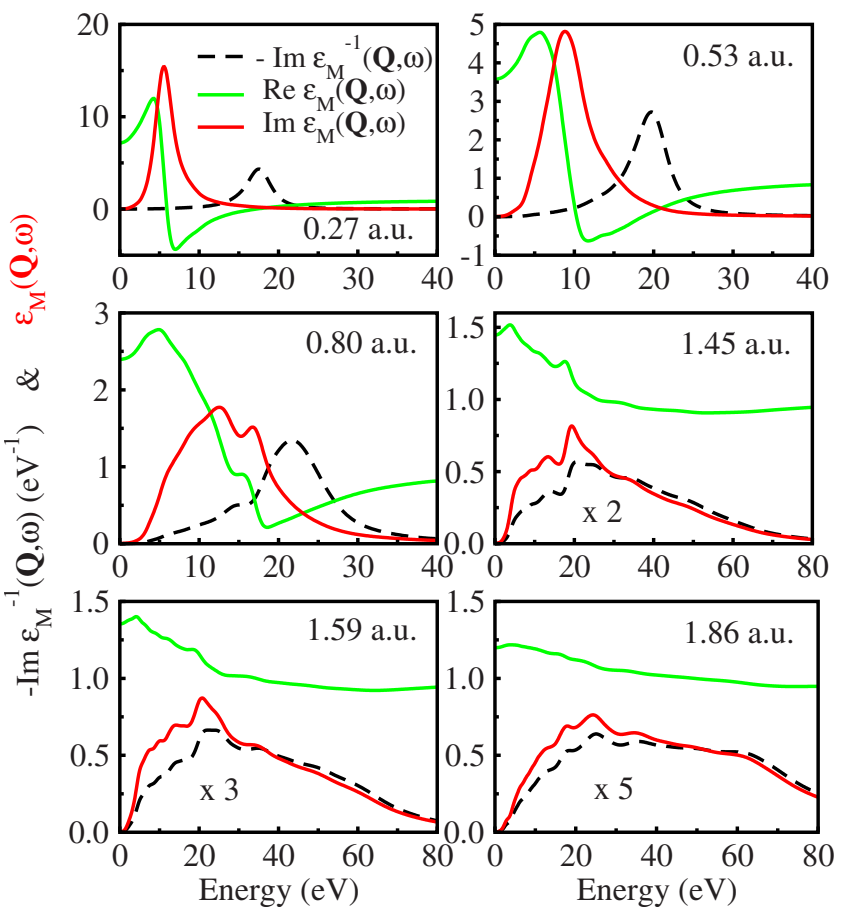

FIG. 20. (Color online) Electron energy-loss and dielectric functions for $\mathbf{Q}$ along [111] as calculated in TDLDA with lifetimes. Note that the imaginary part as well as the loss function have been multiplied by a factor as indicated for better visibility.

understanding that the anisotropy, being a deviation from the behavior of a homogeneous medium like the homogeneous electron gas, is due to band-structure effects and due to the localization of wave functions. The fact that the TDLDA and the full RPA result including CLFE do not show large differences with respect to the anisotropy means that the manybody effects which are simulated by the ALDA do not play any significant role for the anisotropy; its action is isotropic. We mention that the same findings about the anisotropy and its description are valid for $\operatorname{Im} \varepsilon_{\mathrm{M}}(\mathbf{Q}, \omega)$ (not shown).

\section{E. Electron energy-loss function vs $\operatorname{Im} \varepsilon_{M}(Q, \omega)$ - the role of short-range effects}

In Fig. 20 we show the development of $-\operatorname{Im} 1 / \varepsilon_{M}(\mathbf{Q}, \omega)$ and of $\operatorname{Im} \varepsilon_{\mathrm{M}}(\mathbf{Q}, \omega)$ with $\mathbf{Q}$ along [111]. For increasing $\mathbf{Q}$, short-range effects dominate and the screening that causes the difference between $\operatorname{Im} \varepsilon_{\mathrm{M}}(\mathbf{Q}, \omega)$ and $-\operatorname{Im} 1 / \varepsilon_{\mathrm{M}}(\mathbf{Q}, \omega)$ becomes less and less relevant, until finally the two quantities become equal. ${ }^{4,17}$

This is consistent with the fact that the LRC approximation to $f_{\mathrm{xc}}$ yields excellent spectra for a long-wavelength perturbation in extended systems, like for optical $(Q \rightarrow 0)$ absorption in bulk $\mathrm{Si}^{15}$ The long-range component of $\left(v+f_{\mathrm{xc}}\right)$ is important in this case, $\operatorname{Im} \varepsilon_{\mathrm{M}}(\mathbf{Q}, \omega)$ and $-\operatorname{Im} 1 / \varepsilon_{\mathrm{M}}(\mathbf{Q}, \omega)$ are very different, and the effect of the local ALDA is negligible. ${ }^{15}$

By contrast, for localized systems such as semiconductor clusters and nanocrystals, the long-range component of $(v$ $\left.+f_{\mathrm{xc}}\right)$ is much less important. On the other hand, local-field or depolarization effects which enter via the off-diagonal el- 
ements of the dielectric matrix, are strong. They are produced [cf. Eq. (6)] by the Coulomb kernel without its longrange contribution, $v-v_{0}$. The CLFE are the predominant effect to produce the difference between independent-particle spectra (i.e., RPA without CLFE) and spectra calculated in, e.g., TDLDA. The effect of the ALDA kernel, however, is very small, the difference between absorption spectra calculated using the (full) RPA and the TDLDA for clusters such as $\mathrm{Si}_{5} \mathrm{H}_{12}$ or larger nanocrystals such as $\mathrm{Si}_{83} \mathrm{H}_{108}$ are small. ${ }^{17,60}$

Turning now back to the IXS experiment, we have a third situation: an extended crystal but a perturbation that corresponds to a rather short wavelength. With increasing $\mathbf{Q}$, the long-range component of $v$ becomes less and less important so that $\operatorname{Im} \varepsilon_{\mathrm{M}}(\mathbf{Q}, \omega)$ and $-\operatorname{Im} 1 / \varepsilon_{\mathrm{M}}(\mathbf{Q}, \omega)$ finally become equal as shown in Fig. 20. Likewise, the LRC approximation to $f_{\mathrm{xc}}$ produces less and less effects, as can be seen in Fig. 8. The CLFE are very important in some parts of the spectra, in particular for the coupling of the resonant and the antiresonant parts of the polarizability. ${ }^{30}$ However, they remain much less strong than in the case of strongly localized systems because of the much weaker inhomogeneity of the extended crystal.

We are, therefore, in a regime where the short-range effects of $v+f_{\mathrm{xc}}$ are predominant. In this way is can be understood that the local approximation to the exchangecorrelation kernel fares well in the description of the exchange-correlation effects in the electronic response of the IXS experiment.

\section{F. Description of the silicon $L_{2,3}$ edge by the TDLDA pseudopotential calculation}

The measurements for the largest momentum transfers presented in Fig. 1 show the silicon $L_{2,3}$ edge at $99.6 \mathrm{eV}$. (Only the $\mathbf{Q}=1.86$ a.u. along [111] and $\mathbf{Q}=1.86,2.12$, and 2.39 a.u. along [111] exhibit the edge. The other measurements have been carried out only up to just below the edge.) The edge is due to excitations of the outer core electrons. The measurement of this spectral region is known as x-ray Raman spectroscopy, ${ }^{31}$ and the spectra at low momentum transfers are equivalent to soft x-ray absorption spectra of the corresponding core electrons.

Core excitations have been modeled by a number of different approaches. Soininen et al. use the Bethe-Salpeter equation based on a description of the respective orbitals using a pseudopotential approach for the valence states and an atomic Hartree-Fock approach for the core states. ${ }^{61}$ Other approaches are the real-space multiple-scattering method, developed likewise by Soininen et al., ${ }^{62}$ core-hole pseudopotentials, and the $Z+1$ method. ${ }^{63}$ Work in particular on the silicon $L_{2,3}$ edge has been done by Sternemann et al. ${ }^{64}$

Despite the success of these methods dedicated to the description of the core excitations, the question remains as to how the standard TDDFT pseudopotential calculations fare for these excitations. This is particularly important for materials where the core excitation is less clearly separated from the valence response, as, for instance, in the case of $\mathrm{Ge}$ or in transition metals where the excitation of semicore electrons is much closer to the valence spectrum. ${ }^{20,65}$

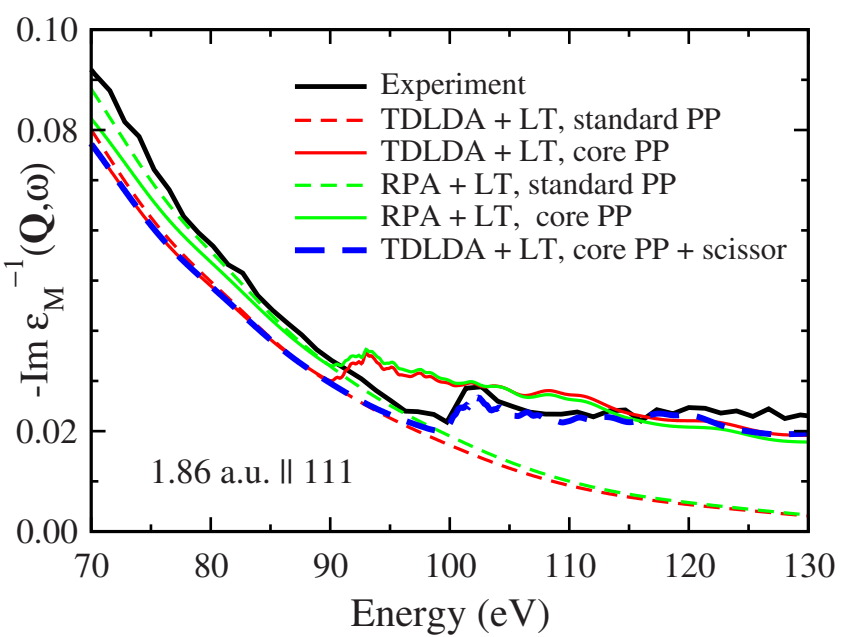

FIG. 21. (Color online) High-energy region of the spectrum for $\mathbf{Q}=1.86$ a.u. in the [111] direction, including the silicon $L_{2,3}$ edge. All the calculations are with the lifetime effects included. "Standard" refers to the calculations with only the $3 s$ and $3 p$ valence electrons while "core" refers to the additional inclusion of the outer core states $2 s$ and $2 p$ in the valence.

In order to test the description of the high-energy part of the spectrum by the TDLDA pseudopotential calculation, we created a pseudopotential including the $2 s$ and $2 p$ electrons in the valence. A detailed test of this pseudopotential is described elsewhere. ${ }^{66}$ The inclusion of the outer core states is computationally rather expensive because the required cutoff energy for the expansion of the core wave functions is high. We use 256 shifted $\mathbf{k}$ points for these calculations. Small differences in the loss function at low energies due to the core polarization will be discussed elsewhere. ${ }^{67}$

The results showing the high-energy region including the silicon $L_{2,3}$ edge are shown in Fig. 21. As no calculated lifetimes for the core states appear to be available, we use the experimental value of approximately $0.2 \mathrm{eV} .^{68}$ A slightly smaller value has been reported in Ref. 69. However, the edge has been found to be very insensitive to the precise value. The inclusion of the lifetimes is important for the visibility of the edge. Without the lifetimes, the calculated spectra are so spiky in this region that the edge is not clearly discernible (not shown). The good visibility in the spectra with lifetimes is due to the fact that the lifetime inclusion according to Eq. (10) describes a rather strong broadening for the transitions from the true valence states into the very high conduction states. This produces the smooth behavior seen in Fig. 21 for the calculations which include only the true valence states. The edge, however, is due to transitions from the outer core states into the lowest conduction states. Both have very long lifetimes. Accordingly, the transitions are only weakly broadened and the edge appears as the sharp feature on the smooth tail of the (true) valence response.

There is essentially no difference between the RPA and the TDLDA spectra of the edge. Moreover, the RPA spectra with and without CLFE do not differ in this region (not shown). In particular, the tail of the valence spectrum remains almost unchanged. This means that the transitions from the outer core states are not appreciably mixed with those from the valence by the local-field effects. 
The energetic position of the edge is underestimated by about $9 \mathrm{eV}$, which corresponds to the overestimation of the energy of the outer core states in the band structure (not shown). This stands in contrast to the results of the response of the valence electrons which we show in the present paper but in agreement with the findings of Gurtubuy et al. who find for transition metals that the response of semicore states is much less well described than that of the valence electrons. ${ }^{20}$

This leads to the question as to what is the reason of the energy difference. First of all, it is possible that the static LDA used for the ground-state calculation does not describe the strongly localized outer core states well. However, the spectra should contain also the self-energy effects, i.e., the energy corrections due to excitation at the one-quasiparticle level, as well as the electron-hole interaction, both simulated by $f_{\mathrm{xc}}$. However, the TDLDA kernel does not change the energy of the edge at all, as can be seen from the comparison with the RPA result. The energy of about $9 \mathrm{eV}$ which is needed to shift the edge to the experimental position corresponds roughly to the self-energy correction found by Rohlfing et al. using a self-consistent $G W$ calculation. ${ }^{70}$ The exciton binding energy, known to be on the order of $0.3 \mathrm{eV}$ (cf., e.g., Ref. 71), is negligible compared to this shift. After shifting the outer core states down by these $9 \mathrm{eV}$ in the sum of Eq. (7) or (8), respectively, we obtain the result shown in Fig. 21 in good agreement with experiment.

Based on this comparison we conclude that the response of the outer core states can be taken into account, although appearing too low in energy. Agreement with experiment can be obtained applying a shift to the energies of the outer core states that introduces the self-energy effects that are not simulated by the TDLDA kernel for the excitation of the strongly bound outer core electrons, unlike for the valence spectra.

\section{CONCLUSIONS}

We have carried out joint IXS measurements and $a b$ initio calculations to study the dynamic structure factor and the dielectric function of $\mathrm{Si}$. The experiments improved upon older results and the direct collaboration proved invaluable for the comparison with the calculations. Using the KramersKronig relations we extracted the dielectric function from the measurements. This enabled a direct comparison between the dielectric function and the electron energy-loss function. The calculations within the framework of linear-response TDDFT showed that at finite momentum transfer the TDLDA together with lifetime effects yields a very good description not only of the dynamic structure factor and the loss function, but also of the dielectric function, in strong contrast to the situation at zero momentum transfer (optical limit). The TDLDA improves strongly upon the RPA results. For finite momentum transfer, it reproduces significant many-body effects beyond RPA. The results demonstrate that the exact $f_{\mathrm{xc}}$ does produce significant short-range effects that are well represented by the local approximation TDLDA. The form of an exchange-correlation kernel has been presented that includes, in the strict framework of linear-response TDDFT, the same lifetime effects that are described by the modified independent-particle polarizability. The silicon $L_{2,3}$ absorption edge has been described by the pseudopotential calculations, albeit too low in energy so that an additional correction had to be applied. In the valence region, crystal local-field effects have been found to play a very important role for larger momentum transfers, in particular in the energy range between 10 and $30 \mathrm{eV}$. Used within a RPA calculation, the crystal local-field effects are sufficient in order to describe the anisotropy in the response. In analogy with the situation in localized systems, for larger momentum transfers the long-range contribution of the combined Coulomb and exchange-correlation kernel becomes less and less important and the electron energy-loss function becomes equal to the imaginary part of the dielectric function.

\section{ACKNOWLEDGMENTS}

Fruitful discussions with M. Krisch, A. Kaprolat, R. Del Sole, F. Bechstedt, Gy. Vankó, R. Hambach, and Sangheeta Sharma are gratefully acknowledged. The work was supported by the European Union through the NANOQUANTA Network of Excellence under Grant No. NMP4-CT-2004500198 and the Marie-Curie Training Site under Contract No. HPMT-CT-2001-00368, as well as by the ETSF e-I3 (Grant No. INFRA-2007-211956). Beam time was provided by the European Synchrotron Radiation Facility. Computer time was granted by the IDRIS, project GENCI 544, on the NEC SX8. H.-Ch. W. acknowledges support from the European Union through the individual Marie Curie IntraEuropean Grant No. MEIF-CT-2005-025067. J.S. acknowledges partial financial support by CICYT under Grants No. MAT2007-60087 and No. ENE2008-04373.
${ }^{1}$ L. Hedin, Phys. Rev. 139, A796 (1965).

${ }^{2}$ W. Hanke and L. J. Sham, Phys. Rev. Lett. 43, 387 (1979).

${ }^{3}$ G. Onida, L. Reining, R. W. Godby, R. Del Sole, and W. Andreoni, Phys. Rev. Lett. 75, 818 (1995).

${ }^{4}$ G. Onida, L. Reining, and A. Rubio, Rev. Mod. Phys. 74, 601 (2002), and references therein.

${ }^{5}$ E. Runge and E. K. U. Gross, Phys. Rev. Lett. 52, 997 (1984).

${ }^{6}$ E. K. U. Gross and W. Kohn, Phys. Rev. Lett. 55, 2850 (1985).

${ }^{7}$ L. Reining, V. Olevano, A. Rubio, and G. Onida, Phys. Rev. Lett. 88, 066404 (2002).

${ }^{8}$ F. Sottile, V. Olevano, and L. Reining, Phys. Rev. Lett. 91,
056402 (2003)

${ }^{9}$ G. Adragna, Ph.D. thesis, Università di Roma Tor Vergata, 2003.

${ }^{10}$ A. Marini, R. Del Sole, and A. Rubio, Phys. Rev. Lett. 91, 256402 (2003).

${ }^{11}$ R. Stubner, I. V. Tokatly, and O. Pankratov, Phys. Rev. B 70, 245119 (2004).

${ }^{12}$ U. von Barth, N. E. Dahlen, R. van Leeuwen, and G. Stefanucci, Phys. Rev. B 72, 235109 (2005).

${ }^{13}$ V. Olevano, Ph.D. thesis, Università di Roma Tor Vergata, 1999.

${ }^{14}$ X. Gonze, P. Ghosez, and R. W. Godby, Phys. Rev. Lett. 78, 294 (1997). 
${ }^{15}$ S. Botti, F. Sottile, N. Vast, V. Olevano, L. Reining, H.-Ch. Weissker, A. Rubio, G. Onida, R. Del Sole, and R. W. Godby, Phys. Rev. B 69, 155112 (2004).

${ }^{16}$ S. Botti, A. Fourreau, F. Nguyen, Y.-O. Renault, F. Sottile, and L. Reining, Phys. Rev. B 72, 125203 (2005).

${ }^{17}$ F. Sottile, F. Bruneval, A. Marinopoulos, L. Dash, S. Botti, V. Olevano, N. Vast, A. Rubio, and L. Reining, Int. J. Quantum Chem. 102, 684 (2005).

${ }^{18}$ N. Maddocks, R. Godby, and R. Needs, Europhys. Lett. 27, 681 (1994).

${ }^{19}$ J. Z. Tischler, B. C. Larson, P. Zschack, A. Fleszar, and A. G. Eguiluz, Phys. Status Solidi B 237, 280 (2003).

${ }^{20}$ I. G. Gurtubay, J. M. Pitarke, W. Ku, A. G. Eguiluz, B. C. Larson, J. Tischler, P. Zschack, and K. D. Finkelstein, Phys. Rev. B 72, 125117 (2005).

${ }^{21}$ A. G. Marinopoulos, L. Reining, V. Olevano, and A. Rubio, Phys. Rev. Lett. 89, 076402 (2002).

${ }^{22}$ M. Ehrnsperger and H. Bross, J. Phys.: Condens. Matter 9, 1225 (1997).

${ }^{23}$ S. Waidmann, M. Knupfer, B. Arnold, J. Fink, A. Fleszar, and W. Hanke, Phys. Rev. B 61, 10149 (2000).

${ }^{24}$ V. Olevano, M. Palummo, G. Onida, and R. Del Sole, Phys. Rev. B 60, 14224 (1999).

${ }^{25}$ C. Sternemann, S. Huotari, G. Vankó, M. Volmer, G. Monaco, A. Gusarov, H. Lustfeld, K. Sturm, and W. Schülke, Phys. Rev. Lett. 95, 157401 (2005).

${ }^{26}$ Y. Q. Cai, P. C. Chow, O. D. Restrepo, Y. Takano, K. Togano, H. Kito, H. Ishii, C. C. Chen, K. S. Liang, C. T. Chen, S. Tsuda, S. Shin, C. C. Kao, W. Ku, and A. G. Eguiluz, Phys. Rev. Lett. 97, 176402 (2006).

${ }^{27}$ N. Hiraoka, H. Ishii, I. Jarrige, and Y. Q. Cai, Phys. Rev. B 72, 075103 (2005).

${ }^{28}$ S. Galambosi, J. A. Soininen, K. Hämäläinen, E. L. Shirley, and C.-C. Kao, Phys. Rev. B 64, 024102 (2001).

${ }^{29}$ W. Schülke, J. R. Schmitz, H. Schulte-Schrepping, and A. Kaprolat, Phys. Rev. B 52, 11721 (1995).

${ }^{30}$ H.-Ch. Weissker, J. Serrano, S. Huotari, F. Bruneval, F. Sottile, G. Monaco, M. Krisch, V. Olevano, and L. Reining, Phys. Rev. Lett. 97, 237602 (2006).

${ }^{31}$ W. Schülke, Electron Dynamics by Inelastic X-Ray Scattering (Oxford University Press, New York, 2007).

${ }^{32}$ L. Van Hove, Phys. Rev. 95, 249 (1954).

${ }^{33}$ D. Pines and P. Nozieres, The Theory of Quantum Liquids (Benjamin, New York, 1966), Vol. 1.

${ }^{34}$ S. L. Adler, Phys. Rev. 126, 413 (1962).

${ }^{35}$ N. Wiser, Phys. Rev. 129, 62 (1963).

${ }^{36}$ S. Huotari, G. Vankó, F. Albergamo, C. Ponchut, H. Graafsma, C. Henriquet, R. Verbeni, and G. Monaco, J. Synchrotron Radiat. 12, 467 (2005).

${ }^{37}$ R. Verbeni, T. Pylkkänen, S. Huotari, L. Simonelli, G. Vanko, K. Martel, C. Henriquet, and G. Monaco, J. Synchrotron Radiat. 16, 469 (2009).

${ }^{38}$ S. Huotari, B. Boldrini, V. Honkimäki, P. Suortti, and W. Weyrich, J. Synchrotron Radiat. 16, 672 (2009).

${ }^{39}$ K. Sturm, W. Schülke, and J. R. Schmitz, Phys. Rev. Lett. 68, 228 (1992).

${ }^{40}$ Note that one has to be careful with the interpretation of this quantity: whereas for cubic systems at vanishing momentum transfer longitudinal and transverse response coincide, this is no longer true for larger $\mathbf{Q}$ : we are dealing with longitudinal quantities here.

${ }^{41}$ G. Mukhopadhyay, R. K. Kalia, and K. S. Singwi, Phys. Rev. Lett. 34, 950 (1975).

${ }^{42}$ S. Rahman and G. Vignale, Phys. Rev. B 30, 6951 (1984).

${ }^{43}$ A. Fleszar and W. Hanke, Phys. Rev. B 56, 10228 (1997).

${ }^{44}$ B. Arnaud, S. Lebègue, and M. Alouani, Phys. Rev. B 71, 035308 (2005).

${ }^{45} \mathrm{~V}$. Olevano (unpublished).

${ }^{46}$ A. Marini and A. Rubio, Phys. Rev. B 70, 081103(R) (2004).

${ }^{47}$ F. Sottile, M. Marsili, V. Olevano, and L. Reining, Phys. Rev. B 76, 161103(R) (2007).

${ }^{48}$ F. Sottile, Ph.D. thesis, Ecole Polytechnique, 2005.

${ }^{49}$ D. Mearns and W. Kohn, Phys. Rev. A 35, 4796 (1987).

${ }^{50}$ X. Gonze, J. M. Beuken, R. Caracas, F. Detraux, M. Fuchs, G. M. Rignanese, L. Sindic, M. Verstraete, G. Zerah, F. Jollet, M. Torrent, A. Roy, M. Mikami, P. Ghosez, J. Y. Raty, and D. C. Allan, Comput. Mater. Sci. 25, 478 (2002).

${ }^{51}$ J. P. Perdew and A. Zunger, Phys. Rev. B 23, 5048 (1981).

${ }^{52}$ D. R. Hamann, Phys. Rev. B 40, 2980 (1989).

${ }^{53}$ V. Olevano (unpublished), www.dp-code.org

${ }^{54}$ L. X. Benedict, E. L. Shirley, and R. B. Bohn, Phys. Rev. B 57, R9385 (1998).

${ }^{55}$ K. Sturm, Phys. Rev. B 52, 8028 (1995).

${ }^{56}$ We use TDLDA without lifetime broadening in order to avoid any problem arising from the approximate inclusion of the lifetimes.

${ }^{57}$ F. Bruneval, F. Sottile, V. Olevano, R. Del Sole, and L. Reining, Phys. Rev. Lett. 94, 186402 (2005).

${ }^{58}$ P. A. Montano, D. L. Price, A. T. Macrander, and B. R. Cooper, Phys. Rev. B 66, 165218 (2002).

${ }^{59}$ I. G. Gurtubay, W. Ku, J. M. Pitarke, A. G. Eguiluz, B. C. Larson, J. Tischler, and P. Zschack, Phys. Rev. B 70, 201201(R) (2004).

${ }^{60}$ S. Botti, H.-Ch. Weissker, and M. A. L. Marques, Phys. Rev. B 79, 155440 (2009).

${ }^{61}$ J. A. Soininen and E. L. Shirley, Phys. Rev. B 64, 165112 (2001).

${ }^{62}$ J. A. Soininen, A. L. Ankudinov, and J. J. Rehr, Phys. Rev. B 72, 045136 (2005).

${ }^{63}$ R. Buczko, G. Duscher, S. J. Pennycook, and S. T. Pantelides, Phys. Rev. Lett. 85, 2168 (2000).

${ }^{64}$ C. Sternemann, J. A. Soininen, S. Huotari, G. Vankó, M. Volmer, R. A. Secco, J. S. Tse, and M. Tolan, Phys. Rev. B 72, 035104 (2005).

${ }^{65}$ A. T. Macrander, P. A. Montano, D. L. Price, V. I. Kushnir, R. C. Blasdell, C. C. Kao, and B. R. Cooper, Phys. Rev. B 54, 305 (1996).

${ }^{66}$ E. Luppi, H.-Ch. Weissker, S. Bottaro, F. Sottile, V. Veniard, L. Reining, and G. Onida, Phys. Rev. B 78, 245124 (2008).

${ }^{67}$ H.-Ch. Weissker, M. Cazzaniga, L. Caramella, E. Luppi, and L. Reining (unpublished).

${ }^{68}$ A. Cricenti, P. Perfetti, and G. L. Lay, Surf. Sci. 401, 427 (1998).

${ }^{69}$ J. Campbell and T. Papp, At. Data Nucl. Data Tables 77, 1 (2001).

${ }^{70}$ M. Rohlfing, P. Krüger, and J. Pollmann, Phys. Rev. B 56, R7065 (1997).

${ }^{71}$ G. Margaritondo, A. Franciosi, N. G. Stoffel, and H. S. Edelman, Solid State Commun. 36, 297 (1980). 